\title{
Direct-drive target designs as energetic particle sources for the Laser MégaJoule facility
}

\author{
Mauro Temporal ${ }^{\circledR 1}{ }^{\dagger} \dagger$, Benoit Canaud ${ }^{2,3}$ and Rafael Ramis ${ }^{4}$ \\ ${ }^{1}$ École Normale Supérieure Paris-Saclay, Centre Borelli UMR 9010, 4 avenue des Sciences, \\ 91190 Gif sur Yvette, France \\ ${ }^{2}$ CEA, DAM, DIF, F-91297 Arpajon, France \\ ${ }^{3}$ Université Paris-Saclay, CEA, LMCE, 91680 Bruyères-le-Châtel, France \\ ${ }^{4}$ ETSI Aeronáutica y del Espacio, Universidad Politécnica de Madrid, 28040 Madrid, Spain
}

(Received 14 November 2020; revised 2 February 2021; accepted 4 February 2021)

This work aims to analyse the possibility of directly driven imploding spherical targets in order to create a source of energetic particles (neutrons, protons, alphas, tritium and ${ }^{3} \mathrm{He}$ ions) for the Laser MégaJoule facility. $\mathrm{D}^{3} \mathrm{He}$ gas-filled spherical $\mathrm{SiO}_{2}$ glass pellets, irradiated by an absorbed laser intensity of $10^{14} \mathrm{~W} \mathrm{~cm}^{-2}$ or $10^{15} \mathrm{~W} \mathrm{~cm}^{-2}$ have been considered. Depending on the absorbed laser intensity and the amount of the ablated glass layer two distinct regimes have been identified: a massive pusher and an exploding pusher. Both regimes are analysed in terms of hydrodynamics and fast particle spectra. Energetic particle time-resolved spectra are calculated and used to infer ionic temperatures and total areal densities. A parametric study has been performed by varying the shell thickness and target inner radius for both laser absorbed intensities.

Key words: fusion plasma

\section{Introduction}

In the context of the French Laser MégaJoule (LMJ) facility (Cavailler et al. 2004; Ebrardt \& Chaput 2010; Lion 2010; CEA/DAM 2020) energetic particle sources are attractive for diagnosing laser plasma experiments or qualifying new diagnostics. In particular, energetic protons at $14 \mathrm{MeV}$, would be useful for radiography. Moreover, particle sources are widely employed to provide valuable information of several physical mechanisms such as electric and magnetic fields generation (Li et al. 2008; Rygg et al. 2008; Gotchev et al. 2009), proton radiography (Li et al. 2006; Kugland et al. 2012; Rygg et al. 2015), magnetic reconnection (Li et al. 2007; Rosenberg et al. 2015), direct-drive implosion (Canaud \& Garaude 2005; Canaud et al. 2007; Séguin et al. 2012; Brandon et al. 2013, 2014; Craxton et al. 2015; Gus'kov et al. 2018), hydrodynamic instabilities (Kull 1991; Goncharov et al. 1996; Manuel et al. 2012) or hohlraum physics (Lindl 1998; Li et al. 2009, 2010, 2012). In addition, energetic particles would be very useful for stopping power experiments, in order to span the $\mathrm{d} E / \mathrm{d} z$ curves (Li \& Petrasso 1993; Cayzac et al. 2015; Frenje et al. 2015, 2019; Zylstra et al. 2015).

$\dagger$ Email address for correspondence: mauro.temporal@ hotmail.com 
The LMJ is a multiple beam facility (initially designed for 60 quads and currently commissioned for 44 quads each equipped with 4 laser beams) with possibility of versatile employment of beams. One possible way to use it would be the creation of a particle source with a part of the 176 beams and using the rest of the facility to perform dedicated experimental campaign. Developing a monoenergetic particle platform for LMJ would serve to investigate fundamental phenomena on extreme plasmas that can be generated at the LMJ scale.

This study analyses the possibility to design particle sources based on the use of a glass $\mathrm{D}^{3} \mathrm{He}$ gas-filled spherical target (Hicks et al. 2000, 2012; Kurebayashi et al. 2005; Frenje et al. 2015; Rinderknecht et al. 2015; Gatu Johnson et al. 2019). D ${ }^{3} \mathrm{He}$ gas produces a large variety of energetic particles such as protons at a birth energy of $3.03 \mathrm{MeV}$ and 14.68 MeV, Tritons at $1.01 \mathrm{MeV},{ }^{4} \mathrm{He}$ at $3.67 \mathrm{MeV},{ }^{3} \mathrm{He}$ ions at $0.82 \mathrm{MeV}$, and neutrons at $2.45 \mathrm{MeV}$. The selection of the thickness of the $\mathrm{SiO}_{2}$ layer would allow the choice between an exploding pusher regime (Rosen \& Nuckolls 1979; Rosenberg et al. 2016) and a classical inertial confinement massive pusher regime (Atzeni \& Meyer-ter-Vehn 2004).

The paper is organized as follows. Section 2 is devoted to the description of the two regimes. Section 3 characterizes the particle emission and describes the measurement of ionic temperature and areal density that could be inferred from particle spectra. Section 4 presents parametric variations made on the shell thickness and inner target radius. Conclusions are drawn in $\S 5$. The results presented in this paper draw general behaviours and cannot be taken strictly. They must be used as general guide in order to design specific sources. In addition, as LMJ is not really dedicated to direct drive, this work must be completed by a two/three-dimensional analysis (Canaud et al. 2002, 2004, 2007; Temporal \& Canaud 2009, 2011; Temporal et al. 2011, 2014a,b,c,d, 2015a,b,c; Canaud \& Temporal 2010; Ramis et al. 2014, 2019) that can lead to different quantitative results. Nevertheless, the qualitative trend remains valid.

\section{Hydrodynamics of the capsule implosions}

\subsection{Two reference cases}

Direct-drive spherical capsules composed of an inner core of radius $r=450 \mu \mathrm{m}$ filled by $\mathrm{D}^{3} \mathrm{He}$ gas at a density of $2.5 \mathrm{mg} \mathrm{cm} \mathrm{cm}^{-3}$ (18 atm) and surrounded by a solid $\mathrm{SiO}_{2}$ shell $\left(\rho=2.2 \mathrm{~g} \mathrm{~cm}^{-3}\right)$ of thickness $\Delta$ are considered. The hydrodynamics evolution of the capsule directly irradiated by a laser beam has been calculated by using the MULTI-IFE code (Ramis, Schmalz \& Meyer-Ter-Vehn 1988; Ramis \& Meyer-ter-Vehn 2016). In these calculations the laser light $(3 \omega, \lambda=0.35 \mu \mathrm{m})$ propagates along the radial direction neglecting refraction and the absorption is modelled by inverse-bremsstrahlung. The code assumes electron and ions plasma temperatures, Spitzer heat conduction (Spitzer 1962) harmonically limited $(10 \%)$ to the free streaming limit, multi-group radiation transport and tabulated equations of state. The laser intensity considered here is the intensity absorbed along direct and return centred ray paths (one-dimensional ray-tracing).

It is worth noting that the simplified physics included in our modelling (e.g. refraction, cross-beam energy transfer and resonant absorption have been neglected) or a different choice of some parameters (e.g. the flux limiter) can influence the results.

In order to illustrate the two different regimes, we consider the following two baseline spherical target designs.

(a) A first design characterized by a thick $\mathrm{SiO}_{2}$ shell of $\Delta=3 \mu \mathrm{m}$ is irradiated by a relatively low laser intensity $I_{\mathrm{abs}}=10^{14} \mathrm{~W} \mathrm{~cm}^{-2}$. This case concerns the massive pusher regime (see $§ 2.2$ ). 
(a)

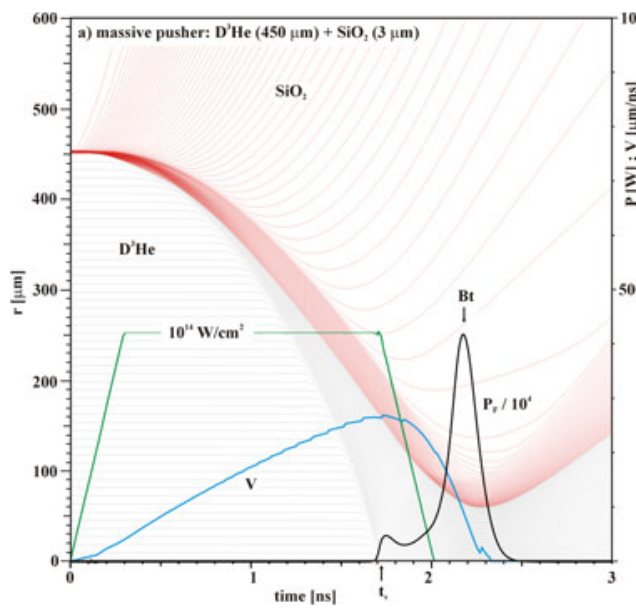

$(b)$

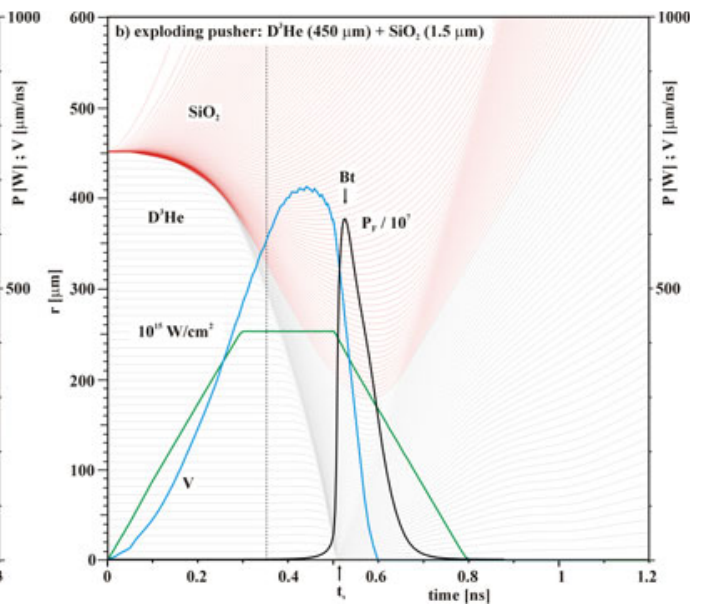

FIGURE 1. Flux chart for $(a)$ the massive pusher case and $(b)$ the exploding pusher case. The Lagrangian cells of the $\mathrm{SiO}_{2}$ shell have been drawn in red, whereas the cells of the $\mathrm{D}^{3} \mathrm{He}$ fuel are drawn in grey. The absorbed laser intensity, the produced fusion power $\left(P_{F}\right)$ and the implosion velocity $(V)$ are shown as a function of time.

(b) A second design made of a thin $\mathrm{SiO}_{2}$ shell of $\Delta=1.5 \mu \mathrm{m}$ is irradiated by a higher laser intensity $I_{\mathrm{abs}}=10^{15} \mathrm{~W} \mathrm{~cm}^{-2}$. We call this case the exploding pusher regime (see $§ 2.2$ ) even if it is not fundamentally the case. Indeed, energy absorption is not volumetric throughout the shell as in a conventional $(1 \omega)$ hot-electron-driven exploding pusher.

The laser pulse shape is chosen to be trapezoidal; the laser intensity grows linearly in 300 ps up to the maximum and remains constant until the time of first shock collapse to the capsule centre $\left(t_{s}\right)$, then it decreases linearly to zero in the next $300 \mathrm{ps.}$

The temporal evolution of the Lagrangian cell positions is shown in figure 1 . The red cells concern the $\mathrm{SiO}_{2}$ shell and the grey cells describe the evolution of the fuel gas. The laser pulse (in green in figure 1) irradiates the external part of the capsule that is heated at high temperature. The $\mathrm{SiO}_{2}$ shell evaporates and gas blow-off generates a strong shock wave at high pressure that moves inward. This shock wave propagates through the shell, crosses the gas and collapses to the target centre at the time $t_{s}$. When the shock reaches the gas-shell interface and enters the gas fuel, a rarefaction wave propagates backwards in the compressed shell up to the ablation where the ablation pressure is generated by the laser absorption. This accelerates the whole shell and the implosion velocity increases up to a maximum. This corresponds to the acceleration phase. The implosion velocity $(V)$ is calculated as $V=\left[\left(\sum m_{i} v_{i}^{2}\right) /\left(\sum m_{i}\right)\right]^{1 / 2}$, where $m_{i}$ and $v_{i}$ are the mass and velocity of imploding cells $\left(v_{i}<0\right)$. This velocity is related to the average value of the kinetic energy and is shown by the blue curve in figure 1 .

After the shock collapse, the laser intensity decreases and a return shock travels back through the target. The implosion velocity reaches a maximum. The deceleration begins and stands for a shorter time than the acceleration phase. At the minimum gas-shell interface radius $r_{\min }$, the implosion stagnates and the shell velocity vanishes. During the deceleration, the ionic temperature rises up to a few kiloelectronvolts and fusion reactions occur. The fusion power increases during this time and achieves a maximum peak power. In some cases, the collapse of the first shock could produce a first small peak of 
fusion power. A second is due to confinement of the fuel. The peak power is achieved at the bang time $\left(B_{t}\right)$. The convergence ratio $(\mathrm{CR})$ of the implosion is defined as the initial capsule radius $r=450 \mu \mathrm{m}$ divided by the minimum radius of the compressed fuel $\left(r_{\min }\right)$ : $\mathrm{CR}=r / r_{\min }$.

As we are designing an energetic particle source in a non-dedicated direct-drive configuration, the implosion can be very sensitive to non-uniformities of irradiation with a potential degradation of particle production (Ramis et al. 2019). Nevertheless, before the arrival of the first shock wave at the target centre, part of the neutrons $n_{s}=n(t<$ $t_{s}$ ) is generated early in the implosion time (details are discussed in $§ 4.2$ ), and their production would not be affected by potential degradations due to laser non-uniformities and hydrodynamic instabilities. Thus, these neutrons provide an estimate of a minimum yield (a safety yield) that can be achieved on LMJ, whatever the uniformity of irradiation is, under reasonable conditions. Indeed, in strongly deteriorated illumination conditions such an estimation fails to predict any safety yield.

\subsection{Differentiating massive pusher and exploding pusher regimes}

In case (a), the first shock wave arrives at the capsule centre at the time $t_{s} \approx 1.7$ ns and the laser stops 300 ps after, at 2 ns. The laser never breaks through the shell that stays massive and compresses the fuel at least 500 ps after the first shock collapse. This is the massive pusher regime. During this delay, the shell kinetic energy is converted into internal energy. The particle emission is mainly done during this time-interval. A maximum fusion power of $P_{\mathrm{F}}=4.2 \times 10^{6} \mathrm{~W}$ is reached at the bang time $B_{t} \approx 2.2 \mathrm{~ns}$, the total number of neutrons is $n=7.5 \times 10^{8}$, the produced fusion energy is $E_{F}=0.9 \mathrm{~mJ}$, the maximum implosion velocity is $V=268 \mu \mathrm{m} \mathrm{ns}^{-1}$ and $\mathrm{CR}=7.6$.

In case (b), owing to higher laser intensity the laser energy deposition is larger. Consequently, the laser light breaks through the shell and penetrates into the $\mathrm{D}^{3} \mathrm{He}$ shell at the time $t_{L}=352 \mathrm{ps}$ shown by the vertical dotted line in figure 1. This generates the explosion of the $\mathrm{SiO}_{2}$ shell: this is the exploding pusher regime. In this case, the implosion is faster, the first shock wave arrives at the capsule centre at the time $t_{s}=0.51$ $\mathrm{ns}$, the fusion power reaches a maximum of about $P_{F}=6.2 \times 10^{9} \mathrm{~W}$, the total number of neutrons provided by the DD reactions is $n=8.5 \times 10^{10}$, the fusion energy is $E_{F}=537$ $\mathrm{mJ}$, the maximum implosion velocity is $V=687 \mu \mathrm{m} \mathrm{ns}^{-1}$ and $\mathrm{CR}=2.5$. After the laser breakthrough, a fraction $(5.7 \%)$ of the total laser energy $(12.8 \mathrm{~kJ})$ is directly deposited into the $\mathrm{D}^{3} \mathrm{He}$ fuel; nevertheless, this small laser energy deposition does not affect the whole dynamic of the implosion. The particle's emission is mainly done during the returning of the shock wave until the shock meets the gas-shell interface. The shell does not confine the fuel that is blown off when the laser breaks through it. In this case, the inertial confinement is only due to the imploding fuel itself. A set of characteristic parameters of these two references cases is reported in table 1.

As can be seen, the CR is higher in case (a) than in case (b). The first case (a) involves less laser energy and benefits more the compression neutrons with respect to the first shock neutrons $\left[n / n_{s} \approx 341\right.$, where $n_{s}$ are the neutrons generated before the arrival of the first shock wave, $\left.n_{s}=n\left(t<t_{s}\right)\right]$. The stagnation stands longer and maximizes the shell kinetic energy transfer to gas internal energy by inertial confinement. Conversely, the second case (b) prioritizes the transfer of gas kinetic energy to internal gas energy. Less inertial kinetic energy is converted in gas internal energy and the major part of the neutrons is produced when the shock returns back to the exploded shell $\left(n / n_{s} \approx 24\right)$.

In order to highlight the differences between these two regimes, a detailed analysis of the hydrodynamic evolution has been performed. It shows that in the first case (a) the laser ablation of the $\mathrm{SiO}_{2}$ shell creates an expanding low-density corona and induces a 


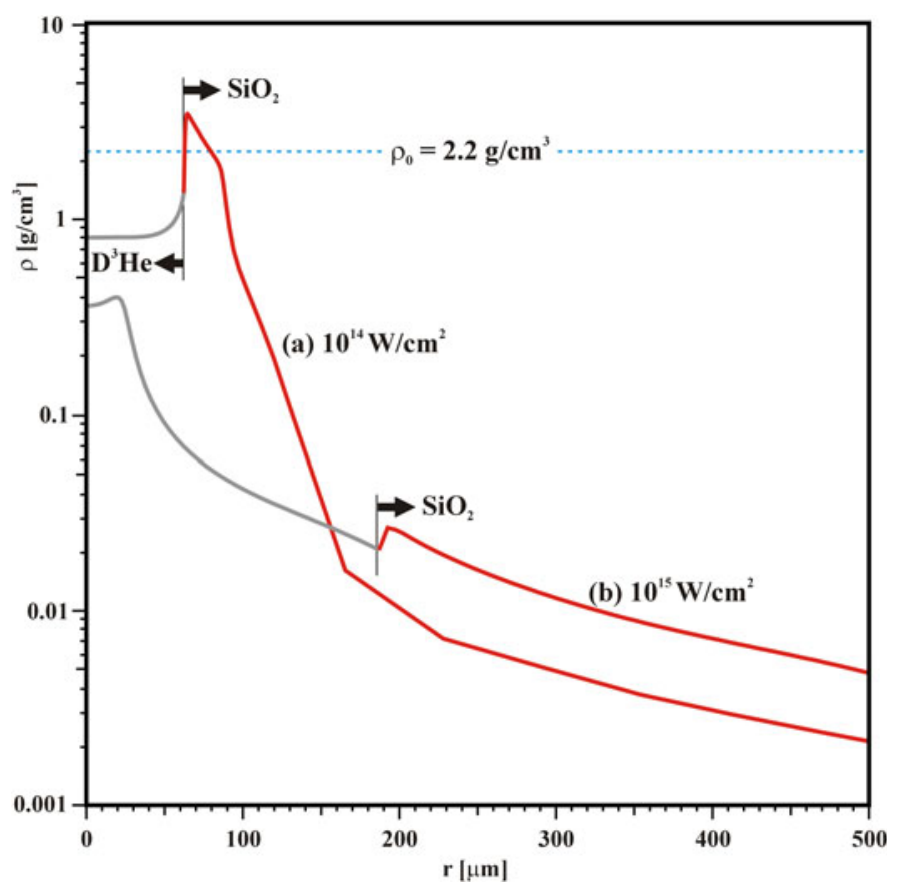

FIGURE 2. Density profiles evaluated at the bang time as a function of the capsule radius.

shock wave that compresses the non-ablated $\mathrm{SiO}_{2}$ mass. The last generates a dense shell that confines the inner $\mathrm{D}^{3} \mathrm{He}$ fuel. Differently, in the second case (b) a large part of the $\mathrm{SiO}_{2}$ mass is ablated preventing the formation of a high-density shell. These different behaviours are shown in figure 2 that presents the density profiles $\rho(r)$ of the two cases (a) and (b) evaluated at the bang time $\left(B_{t}\right)$.

The grey parts of the curves represent the density in the $\mathrm{D}^{3} \mathrm{He}$ fuel, whereas the red parts correspond to the densities in the $\mathrm{SiO}_{2}$. The horizontal dashed line shows the initial $\mathrm{SiO}_{2}$ density $\rho_{0}=2.2 \mathrm{~g} \mathrm{~cm}^{-3}$. It is possible to see that in case (a) of the low laser intensity $I_{\mathrm{abs}}=10^{14} \mathrm{~W} \mathrm{~cm}^{-2}$ a shell with a $\rho_{\max }>\rho_{0}$ confines the fuel, whereas in case (b) the confining high-density shell is not produced and the maximum $\mathrm{SiO}_{2}$ density is always smaller than the initial value $\rho_{0}$. This suggests a criterion to discriminate between the massive pusher regime and the exploding pusher regime, for which $\rho_{\max }>\rho_{0}$ and $\rho_{\max }<\rho_{0}$ respectively.

In the exploding pusher regime (b), the laser light enters into the $\mathrm{D}^{3} \mathrm{He}$ fuel at the time $t_{L}=0.35 \mathrm{~ns}$. In order to evaluate the effect of the laser interaction with the fuel, a calculation has been performed neglecting the laser energy deposition into the $\mathrm{D}^{3} \mathrm{He}$ fuel, hereafter referred as case $\left(b^{*}\right)$. Figure 3 shows the comparison between the mass-average ionic $\left\langle T_{i}\right\rangle$ and electronic $\left\langle T_{e}\right\rangle$ temperatures in the fuel for both cases (b) and (b*) as a function of time. The vertical line shows the time $t_{L}=0.35 \mathrm{~ns}$. As can be seen, the average ionic temperatures are almost the same in both cases even if $\left\langle T_{i}\right\rangle$ is slightly higher whereas the electronic temperature $\left\langle T_{e}\right\rangle$ is larger in case (b). Moreover, it is found that the fusion power $\left(P_{F}\right)$ is slightly higher when neglecting the laser energy deposition into the fuel (b*) and the total nuclear fusion energy increases by $2.2 \%$ from the $E_{F}=537 \mathrm{~mJ}$ of the reference case (b) to $E_{F}=549 \mathrm{~mJ}$ for case (b*). 


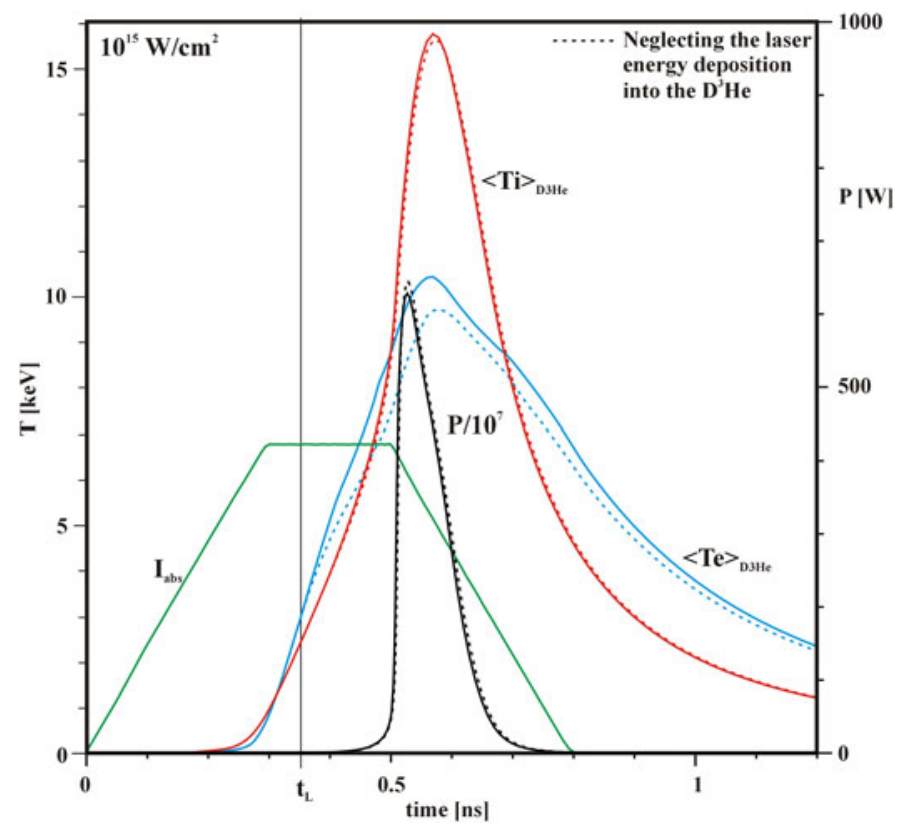

FIGURE 3. Temporal evolution of the average ionic and electronic fuel temperature as a function of the time. The continuous line refers to the exploding pusher case (b) and the dotted line refers to the same case but neglecting the laser energy deposition into the $\mathrm{D}^{3} \mathrm{He}$ fuel. The laser intensity profile is shown in green, the vertical thin line marks the time $t_{L}$.

Therefore, the laser energy deposition in the fuel contributes to a further heating of the fuel mass hampering its compression, and this marginally affects the whole process reducing the total nuclear fusion reactions by a few per cent.

\subsection{Effect of the initial gas density}

In order to evaluate the influence of the initial density of the fuel gas $\left(\rho_{\mathrm{D}^{3} \mathrm{He}}\right)$ in the nuclear yield, some calculations have been performed varying $\rho_{\mathrm{D}^{3} \mathrm{He}}$ from 0.5 to $5 \mathrm{mg} \mathrm{cm}^{-3}$. The results are presented in figure 4 where the total number of produced neutrons $(n)$ is given as a function of the fuel gas density $\rho_{\mathrm{D}^{3} \mathrm{He}}$. The red (blue) full circles refer to the cases at laser intensity $I_{\mathrm{abs}}=10^{15} \mathrm{~W} \mathrm{~cm}^{-2}\left(I_{\mathrm{abs}}=10^{14} \mathrm{~W} \mathrm{~cm}^{-2}\right)$. The number of neutrons $n_{s}=n\left(t<t_{s}\right)$, generated before the arrival of the first shock wave to the target centre, are represented by the red (blue) void circles.

As can be seen, the total number of neutrons is always larger in the exploding pusher cases than in the massive pusher cases. Moreover, the number of neutrons generated before the arrival of the first shock wave at the capsule centre $\left(n_{s}\right)$ is about two orders of magnitude larger for the exploding pusher cases than for the massive pusher cases. In addition, the ratio $n_{s} / n$ is always almost 10 times greater in the exploding pusher than in the massive pusher regime. This leads to the conclusion that, in the context of a non-spherical direct-drive geometry of illumination, an exploding pusher will be a better candidate to create more robust particle sources than a massive pusher.

\section{Time-resolved particle spectra: a tool to diagnose plasma}

\subsection{Description of the particle transport package}

A Monte Carlo package has been implemented in the MULTI code to calculate the transport of the particles generated by the DD, $\mathrm{D}^{3} \mathrm{He}$ and DT nuclear fusion reactions. 


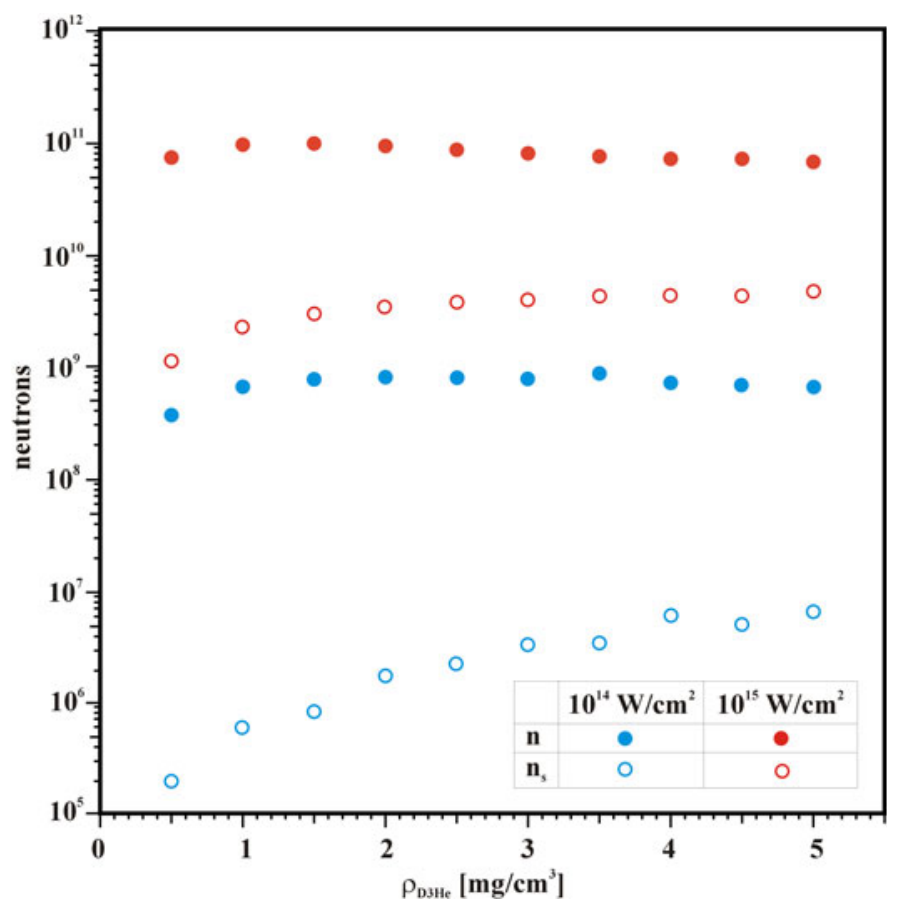

FIGURE 4. Produced neutrons $n$ (full dots) and $n_{s}=n\left(t<t_{s}\right)$ (empty dots) as a function of the fuel density for the laser-absorbed intensity $I_{\mathrm{abs}}=10^{14} \mathrm{~W} \mathrm{~cm}^{-2}$ (blue) and $I_{\mathrm{abs}}=10^{15} \mathrm{~W} \mathrm{~cm}^{-2}$ (red).

The package takes into account the four nuclear fusion reactions, $\mathrm{D}\left({ }^{3} \mathrm{He}, p\right) \alpha(Q=18.35$ $\mathrm{MeV}), \mathrm{D}(\mathrm{D}, n)^{3} \mathrm{He}(Q=3.27 \mathrm{MeV}), \mathrm{D}(\mathrm{D}, p) \mathrm{T}(Q=4.04 \mathrm{MeV})$ and $\mathrm{D}(\mathrm{T}, n) \alpha(Q=17.59$ $\mathrm{MeV}$ ), where the $Q$-value (difference between final and initial masses) represents the available energy in the centre of mass reference frame. All the particles of each kind generated in a time-step by the reactions in a hydrodynamic cell are grouped in $n_{\mathrm{mc}}=100$ macro-particles. The $n_{\mathrm{mc}}$ particles are assumed to be emitted in random directions, thus guaranteeing the isotropy of the source, and moves straightforward through the plasma. In the cases that fusion reactions occur during the laser pulse, the particle energy spectrum can be modified by a laser-generated electric field (Hicks et al. 2000), this effect has not been integrated into our calculations. The Monte Carlo package follows all the particles generated by the fusion reactions. The energy loss of the charged particles $\left({ }^{3} \mathrm{He}, \alpha, p, \mathrm{~T}\right)$ along their path are calculated using the Li-Petrasso stopping power model (Li \& Petrasso 1993, 2015), whereas no interaction of the neutrons $(n)$ with the plasma is considered.

Figure 5 presents the time-resolved spectra of the escaping particles $\left(\alpha,{ }^{3} \mathrm{He}, n, \mathrm{~T}\right.$, $p_{3 \mathrm{MeV}}$, and $p_{14 \mathrm{MeV}}$ ) for the two reference cases (a) and (b). In addition, figure 5 also shows the time-integrated spectra (arbitrary unit) of the generated (black curves) and escaping particles (white curves).

The thin black and white vertical lines indicate the average energy $\left(E_{a}^{i}\right)$ calculated from the time-integrated energy spectra $n_{i}(\varepsilon): E_{a}^{i}=\int n_{i}(\varepsilon) \mathrm{d} \varepsilon / N_{i}$, where $N_{i}$ is the total number of particles $i$.

It is worth noting that the spectra are significantly different in each case. This is mainly due to the interaction of escaping particles with the shell. In case (a), before leaving the target, the particles interact and lose far more energy in the shell than in case (b). 


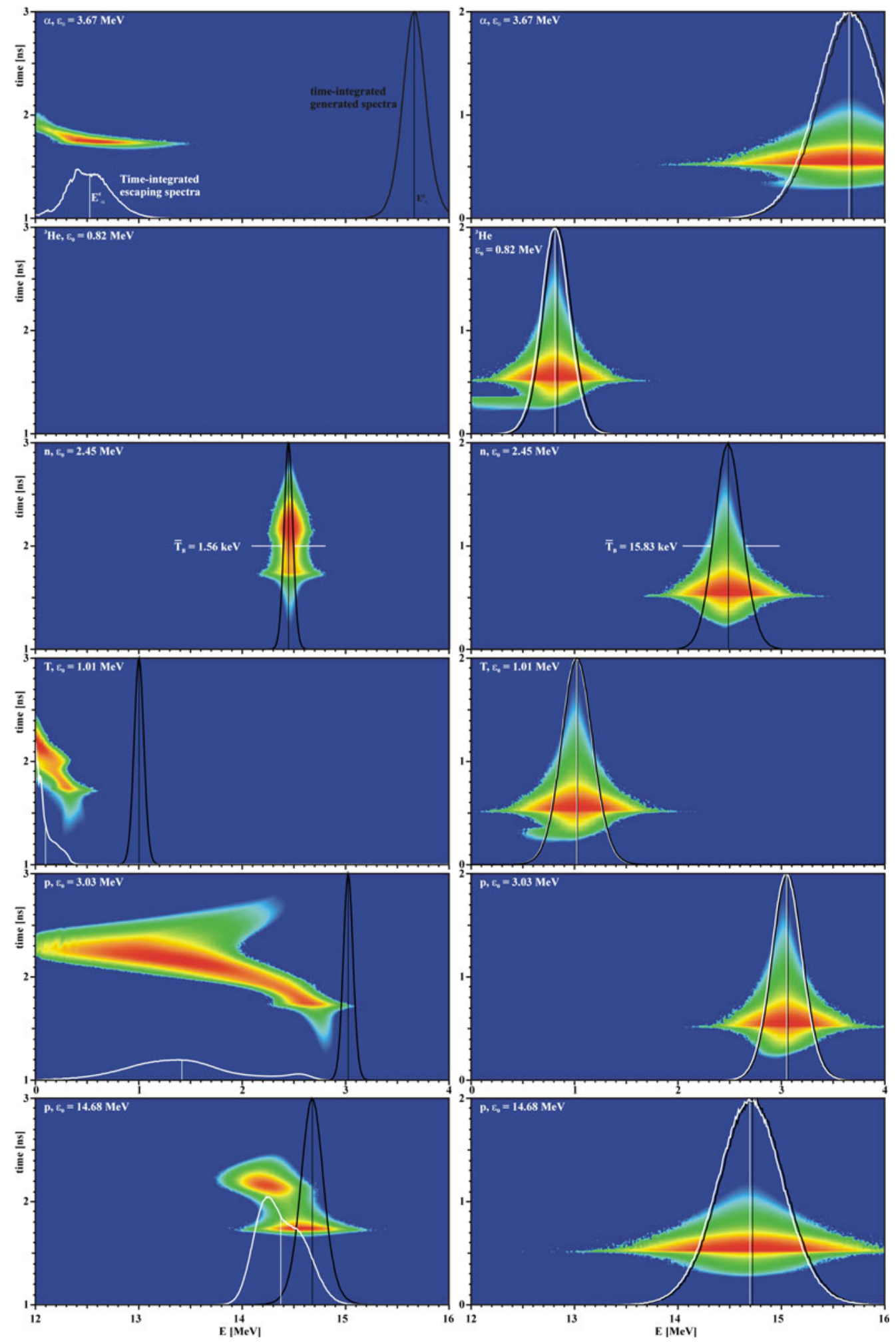

FIGURE 5. Time-resolved spectra of the escaping particles. Left (right) column case at $10^{14} \mathrm{~W}$ $\mathrm{cm}^{-2}\left(10^{15} \mathrm{~W} \mathrm{~cm}^{-2}\right)$. 


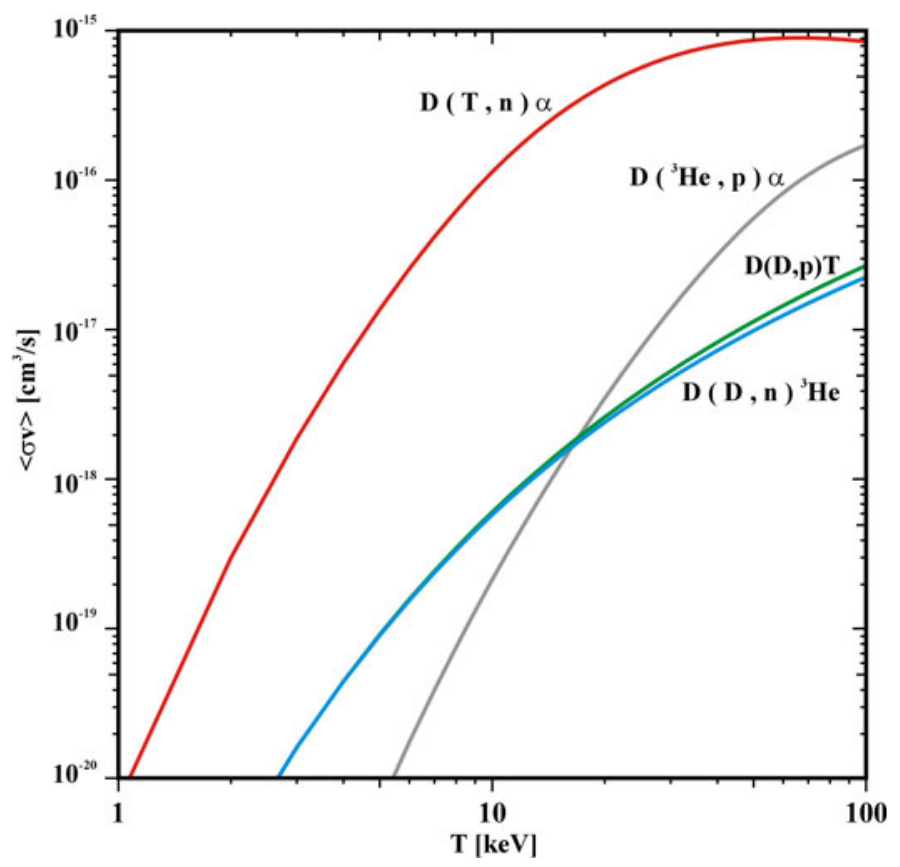

FIGURE 6. Reactivity $\langle\sigma \nu\rangle$ as a function of the plasma temperature $T$.

Indeed, the ${ }^{3} \mathrm{He}$ particles are completely absorbed by the plasma in the massive pusher case (a) whereas they still escape from the plasma in the case of the exploding pusher (b). In addition, the tritium ions are partially stopped inside the plasma in case (a) whereas all of them escape in case (b). It is also worth noting that in case (a) the loss of energy via the Li-Petrasso stopping power model modifies considerably the shape of the time-integrated spectra, providing particles distributions far from Gaussian distributions. However, the relatively small areal density, $\rho R<3 \mathrm{mg} \mathrm{cm}^{-2}$, produced in case (b) avoids a large energy loss and in this case all particles can escape still conserving a Gaussian-like energy distribution.

\subsection{Estimating the ionic temperature by the ratio of particle numbers}

In order to calculate the number of thermonuclear reactions involving $\mathrm{D},{ }^{3} \mathrm{He}$ and $\mathrm{T}$ ions, the code calculates the number densities $n_{\mathrm{D}}, n_{\mathrm{He}}$ and $n_{\mathrm{T}}\left(\mathrm{cm}^{-3}\right)$ of the three reactants in all Lagrangian cells of the fuel. The reaction rate $\mathrm{d} N / \mathrm{d} t=n_{x} n_{a} \sigma v \mathrm{~d} V$, associated with the generic fusion reaction $X(a, b) Y$, depends on the reactivity $\langle\sigma v\rangle\left[\mathrm{cm}^{3} \mathrm{~s}^{-1}\right]$ that is strongly varying with the ionic temperature. Here $n_{x}$ and $n_{a}$ are the number densities of the two reactants $X$ and $a$.

The analytical fit of the reactivities provided by Bosch \& Hale (1992) has been used to generate figure 6 where the four reactivities are shown as a function of the ionic temperature. As is well known, the reactivities of the DD and the $\mathrm{D}^{3} \mathrm{He}$ reactions are about two orders of magnitude lower than that of the DT reaction. Moreover, until the temperature of about $17 \mathrm{keV}$, the $\mathrm{D}^{3} \mathrm{He}$ reactivity is lower than the DD reactivity and it is also worth noting that the reactivity of the $\mathrm{D}(\mathrm{D}, p)$ T reaction is slightly higher than the reactivity of the $\mathrm{D}(\mathrm{D}, n)^{3} \mathrm{He}$ reaction.

The differences between the reactivities, as shown in figure 6 , could be used to estimate an average plasma temperature by time-resolved measurement of the fusion number of 

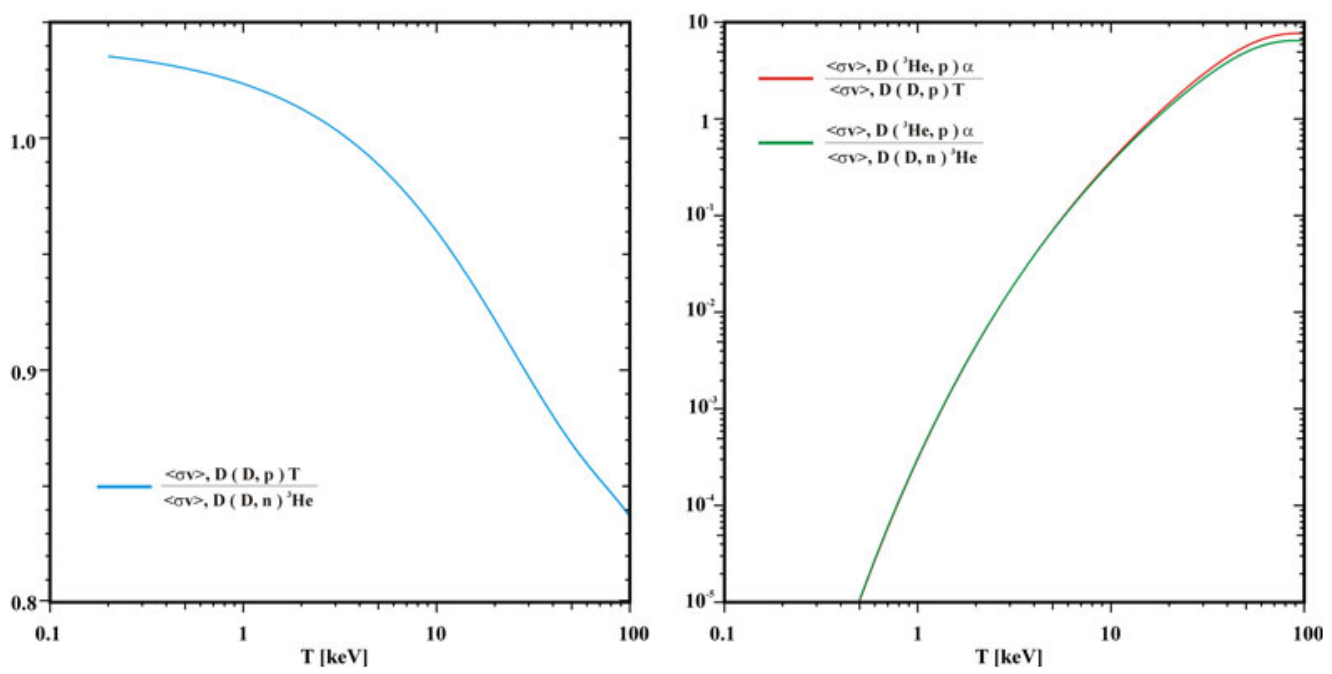

FIGURE 7. Ratios of the reactivities as a function of the plasma temperature $T$.

each reaction. Indeed, the ratio between the generated protons via the $\mathrm{D}(\mathrm{D}, p) \mathrm{T}$ reaction and the number of neutrons via $\mathrm{D}(\mathrm{D}, n)^{3} \mathrm{He}$ reaction are proportional to the ratio of the respective average reactivities:

$$
\frac{\mathrm{d}_{p}^{3 \mathrm{MeV}} / \mathrm{d} t}{\mathrm{~d} N_{n} / \mathrm{d} t}=\frac{\int n_{\mathrm{D}} n_{\mathrm{D}} \sigma v_{\mathrm{D}(\mathrm{D}, p) \mathrm{T}} \mathrm{d} V}{\int n_{\mathrm{D}} n_{\mathrm{D}} \sigma v_{\mathrm{D}(\mathrm{D}, n) \mathrm{He}} \mathrm{d} V}=\frac{\bar{\sigma}_{\mathrm{D}(\mathrm{D}, p) \mathrm{T}}}{\bar{\sigma}_{\mathrm{D}(\mathrm{D}, n) \mathrm{He}}} .
$$

Analogously, assuming $n_{\mathrm{He}} \approx n_{\mathrm{D}}$, it is possible to write the following relations:

$$
\frac{\mathrm{d} N_{p}^{14 \mathrm{MeV}} / \mathrm{d} t}{\mathrm{~d} N_{p}^{3 \mathrm{MeV}} / \mathrm{d} t} \cong \frac{\bar{\sigma}_{\mathrm{D}(\mathrm{He}, p) \alpha}}{\frac{1}{2} \overline{\sigma v}_{\mathrm{D}(\mathrm{D}, p) T}} ; \quad \frac{\mathrm{d} N_{p}^{14 \mathrm{MeV}} / \mathrm{d} t}{\mathrm{~d} N_{n} / \mathrm{d} t} \cong \frac{\overline{\sigma v}_{\mathrm{D}(\mathrm{He}, p) \alpha}}{\frac{1}{2} \overline{\sigma v}_{\mathrm{D}(\mathrm{D}, n) \mathrm{He}}} .
$$

The three reactivity ratios have been calculated using the fit provided by Bosch and Hale and are shown in figure 7 as a function of the plasma temperature $T$.

If we assume to measure the number of neutrons associated with the DD reaction and the number of protons generated by the $\mathrm{DD}$ and $\mathrm{D}^{3} \mathrm{He}$ reactions, the three average reactivity ratios (equal to the ratios of the particle numbers) would provide an estimate of the plasma temperature $\left(T_{B H}\right)$ using the curves in figure 7.

The temporal evolutions of the number of particles, generated by the nuclear fusion reactions, that escape from the target are shown in figure 8 for the two reference cases (a) and (b). In figure 8 the temporal evolution of the total areal density $\rho R$ (dashed lines) is also shown.

For the reference case (b), due to the modest areal density $\rho R<3 \mathrm{mg} \mathrm{cm}^{-2}$, all $\alpha$-particles can escape from the target. Thus, the temporal evolution of the $\alpha$-particles and of the protons at $14 \mathrm{MeV}$, both generated by the $\mathrm{D}\left({ }^{3} \mathrm{He}, p\right) \alpha$ reaction, are the same. However, in the reference case (a) that generates a larger $\rho R$, the $\alpha$-particles are nearly completely retained in the plasma after around $t=2 \mathrm{~ns}$, whereas the protons always exit the target. Similarly, all the tritium ions escape from the target in case (b) whereas they are partially stopped in case (a).

The time integration of the distributions shown in figure 8 provides the total number of particles escaping from the target, and these data have been collected in table 2. 
(a)

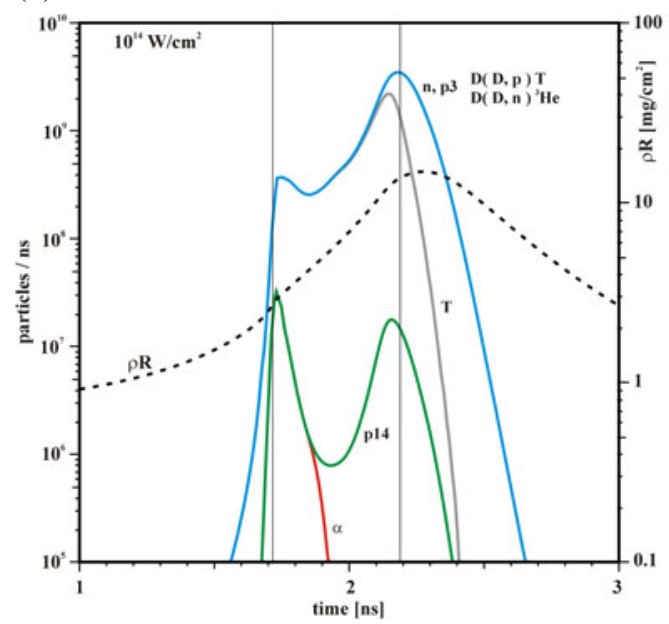

(b)

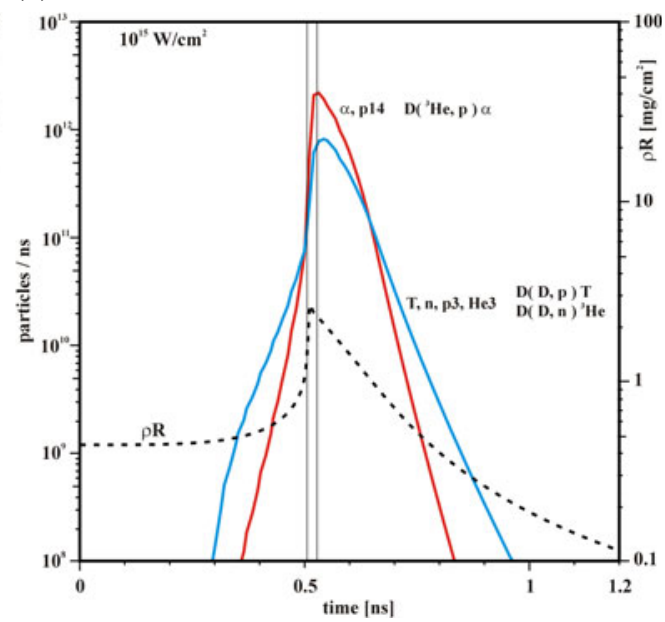

FIGURE 8. Distribution (particles/ns) of the particle number escaping the plasma and the total areal density $\rho R$ as a function of time. The vertical thin lines correspond to the times $t_{S}$ and the bang time $\left(B_{t}\right)$.

$\alpha$

(a) $10^{14} \mathrm{~W} \mathrm{~cm}^{-2}$

(b) $10^{15} \mathrm{~W} \mathrm{~cm}^{-2}$
$1.78 \times 10^{6}$

$1.51 \times 10^{11}$

$$
p_{14 \mathrm{MeV}}
$$

$4.26 \times 10^{6}$

$1.51 \times 10^{11}$
${ }^{3} \mathrm{He}$

0

$8.5 \times 10^{10}$ n

$7.5 \times 10^{8}$

$8.5 \times 10^{10}$

\section{T}

$p_{3 \mathrm{MeV}}$

$4.1 \times 10^{8}$

$7.8 \times 10^{10}$

$7.8 \times 10^{8}$

$7.8 \times 10^{10}$

TABLE 2. Total number of energetic particles escaping from the plasma.
(a) $10^{14} \mathrm{~W} \mathrm{~cm}^{-2}$
(b) $10^{15} \mathrm{~W} \mathrm{~cm}^{-2}$
1.0174
0.937
$5.68 \times 10^{-3}$
1.776
$5.46 \times 10^{-3}$
(a) $10^{14} \mathrm{~W} \mathrm{~cm}^{-2} ; T_{B H}$
$1.54 \mathrm{keV}$
$1.75 \mathrm{keV}$
1.936
(b) $10^{15} \mathrm{~W} \mathrm{~cm}^{-2} ; T_{B H}$
$15.29 \mathrm{keV}$
$16.08 \mathrm{keV}$
$1.74 \mathrm{keV}$
$16.08 \mathrm{keV}$

$N_{p}^{3 \mathrm{MeV}} / N_{n}$

$N_{p}^{14 \mathrm{MeV}} / N_{n}$

$N_{p}^{14 \mathrm{MeV}} / N_{p}^{3 \mathrm{MeV}}$

TABLE 3. Reactivity ratio and their corresponding temperature $T_{B H}$.

Using these time-integrated values the reactivity ratios have been calculated and their corresponding temperatures $T_{B H}$ have been estimated and are reported in table 3 .

If we consider only escaping particles, assuming that a time-resolved measurement of $\mathrm{d} N_{X} / \mathrm{d} t$ can be achieved (as in Sio et al. 2019), a direct measurement of the ionic temperature $T_{B H}$ could be deduced (as shown later in figure 11). Indeed, in Sio et al. (2019), protons and neutrons are simultaneously recorded on a streak camera providing a time-resolved measurement of primary yields with a temporal resolution of $10 \mathrm{ps.} \mathrm{Such}$ a measurement could be very beneficial to infer the hot spot hydrodynamics conditions. 


\subsection{Estimation of the ionic temperature by the Doppler effect}

Let us now consider the nuclear fusion reaction $\mathrm{D}(\mathrm{D}, n)^{3} \mathrm{He}$ and the energy distribution of the generated particles ( $n$ and ${ }^{3} \mathrm{He}$ ). Of course, analogous considerations can be done for any other two-body reactions such as $\mathrm{D}\left({ }^{3} \mathrm{He}, p\right) \alpha, \mathrm{D}(\mathrm{D}, p) \mathrm{T}$ or $\mathrm{D}(\mathrm{T}, n) \alpha$. The total available reaction energy is given by the $Q$-value and the produced particles, $n$ and ${ }^{3} \mathrm{He}$, are emitted at the nominal energies $\varepsilon_{0}=Q\left(m_{n}+m_{\mathrm{He}}-m\right) /\left(m_{n}+m_{\mathrm{He}}\right)$, with $m=m_{n}$ or $m=m_{\mathrm{He}}$. Nevertheless, all particles generated in a Lagrangian cell have an energy spread that depends on the plasma temperature $T$. Following the work of Brysk (1973), it is possible to show that the energy distribution of the generated particles follows the normal Gaussian distribution: $f(\varepsilon)=\mathrm{e}^{-(\varepsilon-\varepsilon)^{2} / 2 \sigma^{2}}$, where $\sigma$ is the standard deviation $\sigma(T)=$ $\sqrt{2 m T \varepsilon /\left(m_{n}+m_{\mathrm{He}}\right)}, T$ the plasma temperature and $\varepsilon$ is the birth energy corrected by the plasma temperature:

$$
\begin{aligned}
\varepsilon & =\frac{m_{n}+m_{\mathrm{He}}-m}{m_{n}+m_{\mathrm{He}}} Q+\left\{\frac{3}{2} \frac{m}{m_{n}+m_{\mathrm{He}}} T+\frac{m_{n}+m_{\mathrm{He}}-m}{m_{n}+m_{\mathrm{He}}}\left[\left(\frac{\pi^{2} e^{4}}{2 h^{2}} \mu\right)^{1 / 3} T^{2 / 3}+\frac{5}{6} T\right]\right\} \\
& =\varepsilon_{0}+B(T) .
\end{aligned}
$$

where $\mu$ is the reduced mass of the reactants. Note that, for a zero plasma temperature $T=0$, one recovers $\varepsilon_{T=0}=\varepsilon_{0}$. Thus, the full width at half maximum (FWHM) of the particle distribution can be correlated to the plasma temperature $T$ at which they have been generated FWHM $=2 \sigma(T) \sqrt{-2 \ln (1 / 2)}$, and shows that the energy dispersion of the produced particles grows proportionally to the square root of the plasma temperature $\sigma \propto T^{1 / 2}$.

The average energy of the generated particles $\varepsilon$ is greater than the nominal energy $\epsilon_{0}$ and the difference $B(T)$ grows with the plasma temperature $T$. In figure 9 the function $\left(\langle\varepsilon\rangle-\varepsilon_{0}=B(T) / \varepsilon_{0}\right.$ is shown as a function of the temperature $T$; it is found that, for a plasma temperature $T<1 \mathrm{keV}$, the increment with respect to $\epsilon_{0}$ is of the order of $0.01 \%$, roughly $0.1 \%$ for $1 \mathrm{keV}<T<10 \mathrm{keV}$, and grows to a few per cent for $T>10 \mathrm{keV}$.

The Monte Carlo package also provides the time-resolved spectra of all generated particles as well as the spectra of the particles that escape the target. The case of the neutrons is peculiar because their interaction with the plasma has been neglected, thus the generated spectra coincide with the transmitted ones. The time-resolved neutron spectra, provided by the $\mathrm{D}(\mathrm{D}, n)^{3} \mathrm{He}$ reaction, are shown in figure 10 for both cases (a) and (b). As a reference, the vertical white lines show the time of the arrival of the first shock at the capsule centre $\left(t_{s}\right)$ and the bang time $\left(B_{t}\right)$. Both neutron spectra are centred on the average energy of the generated particles $\langle\varepsilon\rangle$ that almost coincides with the nominal energy $\epsilon_{0}$ $(2.452 \mathrm{MeV})$ and have a width that depends on the plasma temperature as developed in the Brysk's work. Thus, measuring the distribution dispersion of the neutrons, it is possible to estimate an average Brysk temperature $T_{B}$ at which they have been produced. Indeed, the spectra of figure 10 have been used to calculate the temporal evolution of the dispersion $\sigma(t)$ with which $T_{B}$ is evaluated. Because the average particle energy $\langle\varepsilon\rangle$ also depends on the temperature, the Brysk temperature is estimated by solving iteratively the equation: $T_{B}(t)=\sigma^{2}(t)\left(m_{\mathrm{He}}+m_{n}\right) /\left(2 m_{n} \varepsilon\right)$. The time-resolved Brysk temperature $T_{B}$ (green curve), evaluated as function of time, is shown in figure 10 and almost corresponds to the neutron-weighted ionic $\left(T_{i}\right)$ temperature defined as

$$
T_{n}(t)=\frac{\int n_{\mathrm{D}} n_{\mathrm{D}} \sigma v T_{i} \mathrm{~d} V}{\int n_{\mathrm{D}} n_{\mathrm{D}} \sigma v \mathrm{~d} V},
$$

(red curves) directly provided by the code MULTI. 


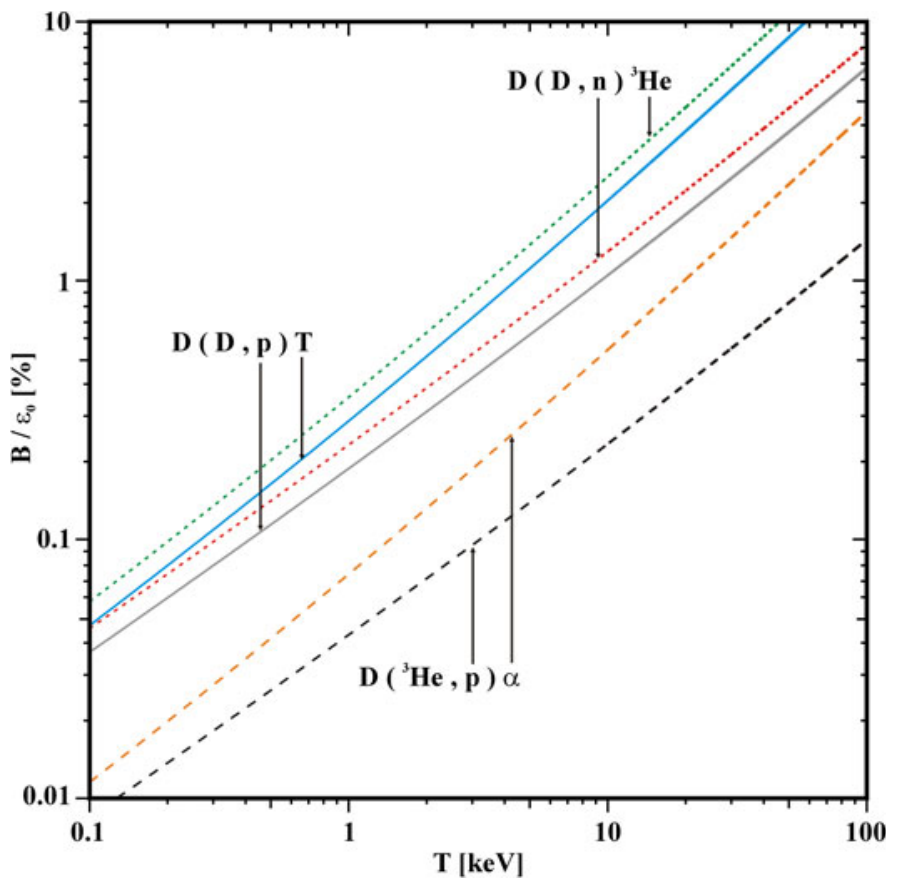

FIGURE 9. Increment of the average birth energy $B(T)$ with respect to the nominal energies $\epsilon_{0}$ as a function of the plasma temperature $T$.

(a)

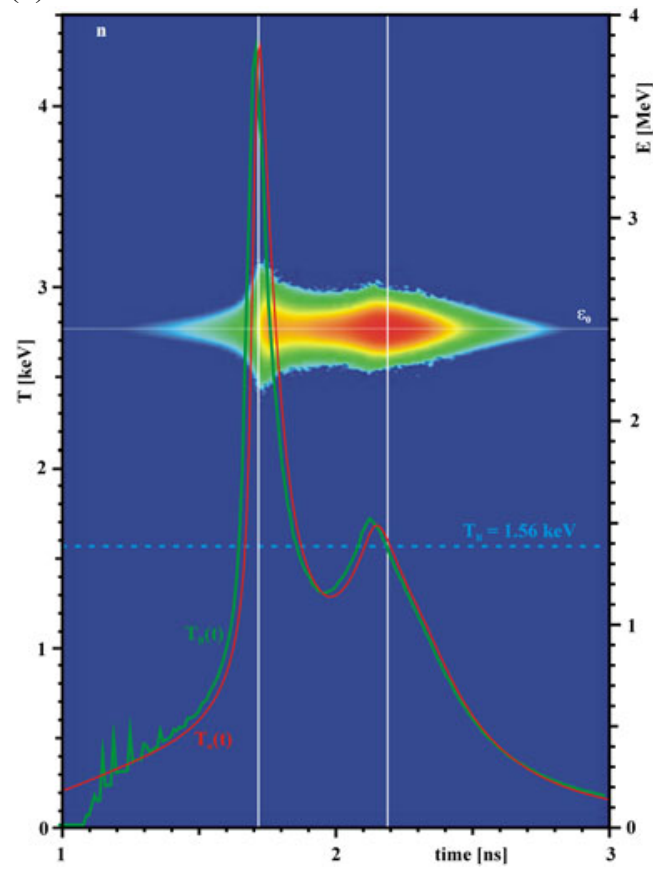

(b)

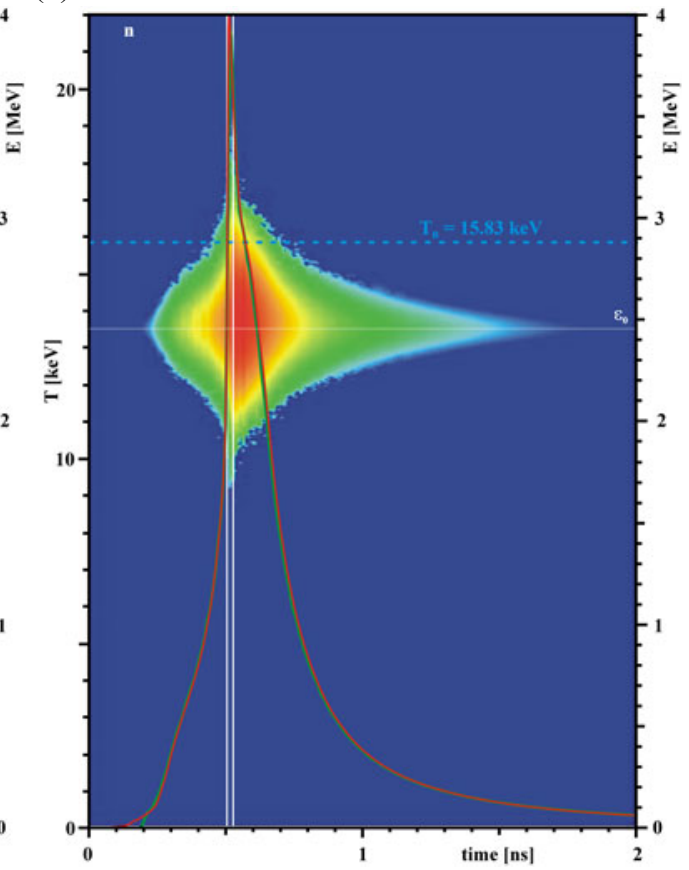

FIGURE 10. Time-resolved neutron spectra (colour scale in arbitrary units). Estimated Brysk temperature $\left(T_{B}\right.$, green curves) and neutron average ionic temperature $\left(T_{n}\right.$, red curves) as a function of time. 

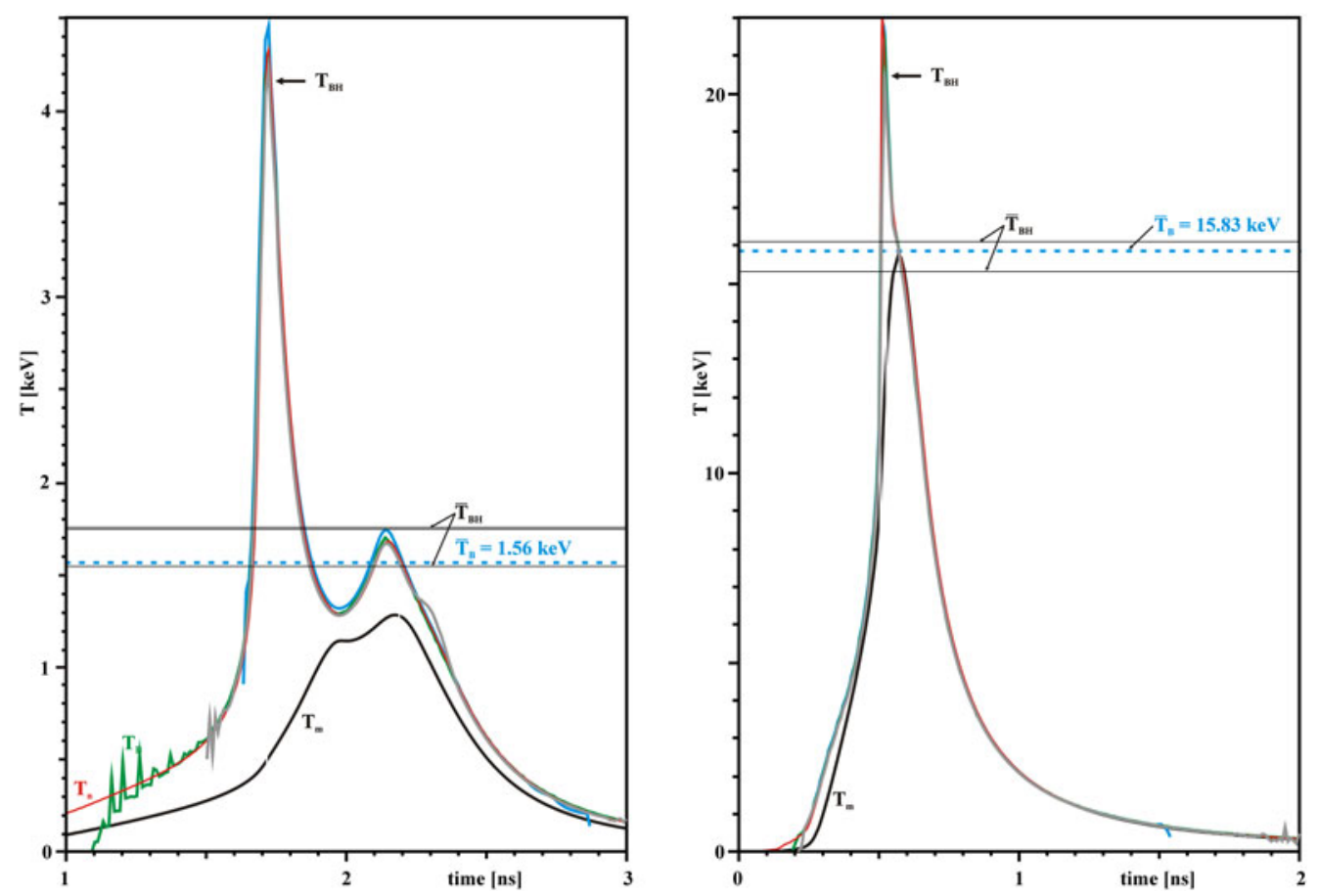

FIgURE 11. Temporal evolution of the Brysk temperature $\left(T_{B}\right.$, green), Bosch and Hale temperatures $\left(T_{B H}\right.$, grey), neutron-average ion temperature $\left(T_{n}\right.$, red) and mass-average fuel temperature $\left(T_{m}\right.$, black) as a function of time. The temperatures $\bar{T}_{B}$ and $\bar{T}_{B H}$, estimated from the corresponding time-integrated quantities, are shown by horizontal lines.

The time-integrated neutrons spectra are usually measured experimentally, and used to estimate the Brysk temperature $\bar{T}_{B}$. For our two references cases (a) and (b), the time-integrated neutron-weighted temperature $\bar{T}_{n}=\iint n_{\mathrm{D}} n_{\mathrm{D}} \sigma v T \mathrm{~d} V \mathrm{~d} t / N_{n}$, where $N_{n}$ is the total number of generated neutrons $N_{n}=\iint n_{\mathrm{D}} n_{\mathrm{D}} \sigma v \mathrm{~d} V \mathrm{~d} t$, provides the temperatures $\bar{T}_{n}=1.55 \mathrm{keV}$ and $\bar{T}_{n}=15.74 \mathrm{keV}$, respectively. These values almost match with the Brysk temperature $\bar{T}_{B}(1.56$ and $15.83 \mathrm{keV})$ calculated using the width of the time-integrated neutron spectra and are shown by the dashed blue lines in figure 10 .

Thus, the particle spectra provide the estimation of four instantaneous temperatures: the Brysk temperature $T_{B}$, deduced by the FWHM of the neutron distribution, and the three $T_{B H}$ temperatures deduced by the ratios of the reactivity based on the fit of Bosch and Hale. In the figure 11 are shown the temporal evolution of these four temperatures, and it is found that all of them match quite well with the neutron-weighted temperature $T_{n}$. These temperatures have been compared also with the mass-average ionic temperature $T_{m}(t)=\int \rho T_{i} \mathrm{~d} V / \int \rho \mathrm{d} V$, where the integral is done over the $\mathrm{D}^{3} \mathrm{He}$ fuel volume. It can be seen that the Brysk temperature, as well as the three $T_{B H}$ estimations, are always larger than the mass average temperature $T_{m}$ (black curve). This is because the temperatures $T_{B}$ and $T_{B H}$ represent an average temperature over the cells where nuclear fusion reactions take place. Low-temperature cells where fusion activity is marginal or missing do not influence them. However, $T_{m}$ is evaluated over the whole $\mathrm{D}^{3} \mathrm{He}$ fuel volume, in which low-temperature cells contribute whatever the nuclear reaction rate is.

In figure 11 the horizontal lines represent the values of $\bar{T}_{B}$ and $\bar{T}_{B H}$ estimated using the time-integrated neutron spectra and the ratios of the total number of escaping 


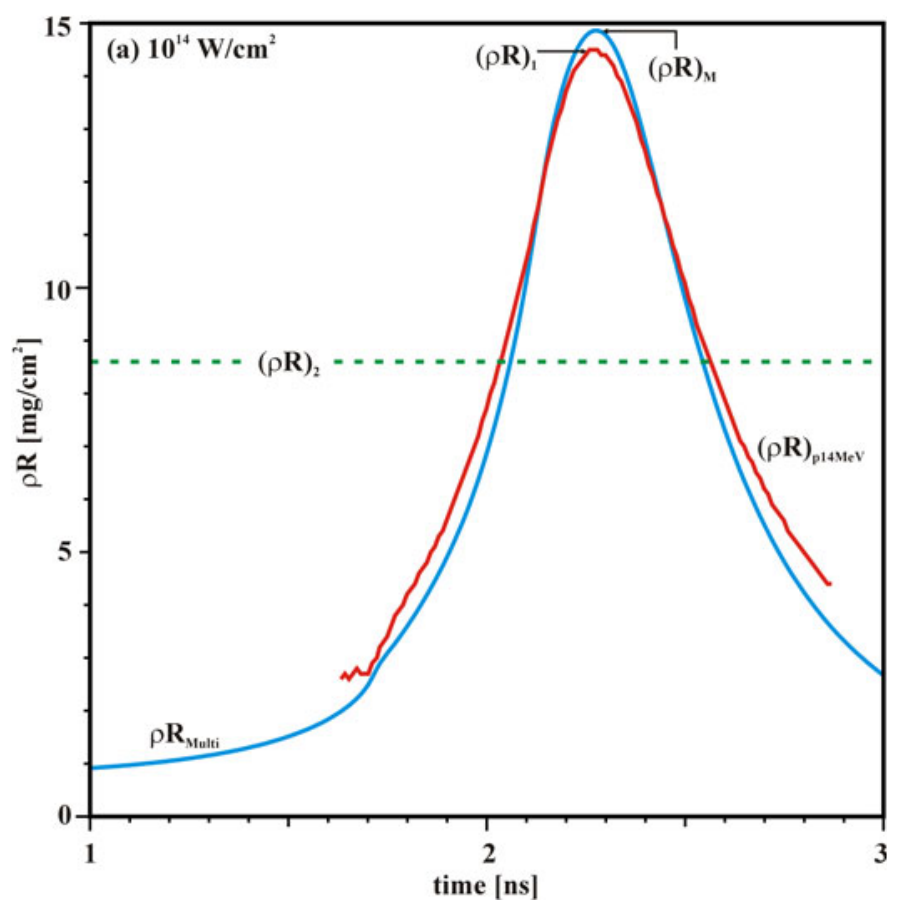

FIGURE 12. Temporal evolution of the areal densities $(\rho R)_{M}$, provided by MULTI code, $(\rho R)_{1}$ estimated from the time-resolved energy loss of the $14 \mathrm{MeV}$ protons. The horizontal dashed green line shows the areal density $(\rho R)_{2}$ calculated using the average energy loss provided by the time-integrated spectra.

particles (table 3). It is found that, in case (b) of the exploding pusher, they almost coincide with the maximum mass-average temperature $T_{\max }=\operatorname{Max}\left(T_{m}\right)=15.73 \mathrm{keV}$; whereas they far exceed the maximum temperature $T_{\max }=1.28 \mathrm{keV}$ found in reference case (a) of the massive pusher.

\subsection{Areal density estimation}

The energy loss of the particles is correlated to the plasma areal density $\rho R$ crossed along their path prior to exiting the target. The $14 \mathrm{MeV}$ protons provided by the $\mathrm{D}^{3} \mathrm{He}$ fusion reactions have been used to estimate the total areal density. The time-resolved spectra shown in figure 5 are used to calculate the temporal evolution of the average proton energy defined by $\bar{\varepsilon}_{p}(t)=\int n_{p}(\varepsilon, t) \mathrm{d} \varepsilon / N_{p}(t)$. It is assumed that such protons have been generated at the Brysk temperature $\bar{T}_{B}$ (provided by the temporal integrated neutrons spectra) with which the average birth energy $\varepsilon$ of the $14 \mathrm{MeV}$ protons is calculated using (3.3). Then the proton energy loss is estimated as $\Delta \varepsilon(t)=\langle\varepsilon\rangle-\bar{\varepsilon}_{p}(t)$. A simplified stopping power $(\mathrm{d} \epsilon / \mathrm{dz})$ has been calculated using the Li-Petrasso model assuming that these protons propagate in a uniform plasma characterized by a density of $\rho=1 \mathrm{~g} \mathrm{~cm}^{-3}$, temperature $\bar{T}_{B}$ and using a constant Coulomb $\operatorname{logarithm} \log (\lambda)=5$.

Finally, it is possible to estimate the total areal density as $\rho R_{p 14 \mathrm{MeV}}(t)=\rho r(t)$, where the protons path $r(t)$ is given by $\Delta \varepsilon(t)=\int_{0}^{r(t)}(\mathrm{d} \varepsilon / \mathrm{d} z) \mathrm{d} r$. In figure 12 (red curves) the temporal evolution of the estimated areal density $\rho R_{p 14 \mathrm{MeV}}(t)$ calculated for the references case (a) is shown. In addition, the total areal density $\rho R_{\text {Multi }}(t)$ directly provided by the MULTI code are shown (blue curve). It is found that the maximum of $\rho R_{p 14 \mathrm{MeV}},(\rho R)_{1}=14.5 \mathrm{mg} \mathrm{cm}^{-3}$ 
grossly correspond to the maximum of $\rho R_{\text {Multi }},(\rho R)_{M}=14.8 \mathrm{mg} \mathrm{cm}^{-3}$. The horizontal green line indicates the areal density $(\rho R)_{2}=8.6 \mathrm{mg} \mathrm{cm}^{-3}$ calculated assuming an energy loss $\Delta \varepsilon(t)=\varepsilon-E_{a}^{p}$, where $E_{a}^{p}$ is the average energy calculated from the time-integrated energy spectra (see the white line in figure 5). It is worth noting that the $14 \mathrm{MeV}$ protons provide a robust estimation of the areal density $\left[(\rho R)_{1} \approx(\rho R)_{M}\right]$ over a wide variation of plasma temperature and density. However, the areal density $(\rho R)_{2}$, estimated using the time-integrated energy spectra, only represents an average areal density and does not reproduce this maximum value.

\section{Parametric study}

In the previous sections it has been shown that the two reference cases, characterized by a capsule radius $r=450 \mu \mathrm{m}$, provide quite different hydrodynamic histories and particle spectra. As commented in $\S 2$, case (a) is characterized by a relatively low laser absorption intensity $I_{\text {abs }}=10^{14} \mathrm{~W} \mathrm{~cm}^{-2}$ and large $\mathrm{SiO}_{2}$ shell thickness $\Delta=3 \mu \mathrm{m}$, whereas in case (b), $I_{\mathrm{abs}}=10^{15} \mathrm{~W} \mathrm{~cm}^{-2}$ and $\Delta=1.5 \mu \mathrm{m}$. In this section we analyse how the capsule performances vary with the capsule radius $(r)$ and shell thickness $(\Delta)$. A parametric study has been performed varying the capsule radius $r$ between 200 and $700 \mu \mathrm{m}$ and the $\mathrm{SiO}_{2}$ shell thickness $\Delta$ between 1 and $5 \mu \mathrm{m}$. A total of 11 radii and 9 thicknesses have been considered. A large number of parameters $\left(E_{\mathrm{abs}}, \mathrm{CR}, V, B_{t}, t_{s}, \rho R_{\mathrm{tot}}, \rho R_{\mathrm{DHe}}, n_{\alpha}, n_{p 14 \mathrm{MeV}}\right.$, $\left.n_{\mathrm{He}}, n_{\mathrm{T}}, n_{3 \mathrm{MeV}}, n_{n}, n_{s}, T_{\max }, T_{B}, \Delta \epsilon\right)$ have been collected and are reported here in the form of contour maps $f(r, \Delta)$.

Facing the huge amount of data and figures, we have separated them by centre of interest.

\subsection{Hydrodynamics of the implosions}

The maximum density $\left(\rho_{\max }\right)$ provided by the $\mathrm{SiO}_{2}$ shell at the bang time has been evaluated, and the contour maps of the ratio $\rho_{\max } / \rho_{0}$ are shown in figure 13 as a function of the capsule radius $r$ and the $\mathrm{SiO}_{2}$ shell thickness $\Delta$. The lightly shaded area corresponds to the cases where the laser-capsule design is in the exploding pusher regime $\left(\rho_{\max } / \rho_{0}<1\right)$. It is shown that for the cases at relatively low laser intensity $I_{\mathrm{abs}}=10^{14} \mathrm{~W} \mathrm{~cm}^{-2}$ only capsules with relatively large radius-to-thicknesses ratio can operate as an exploding pusher. In contrast, for $I_{\mathrm{abs}}=10^{15} \mathrm{~W} \mathrm{~cm}^{-2}$, the massive pusher zone is reduced to small capsule radii and relatively large $\mathrm{SiO}_{2}$ thicknesses. The position of the two reference cases (a) and (b) are shown by the blue and red dots, respectively.

It has been realized that in some cases the laser light breaks through the $\mathrm{SiO}_{2}$ shell at the time $t_{L}$ and penetrates into the fuel. This time can be compared with the arrival time $\left(t_{s}\right)$ of the first shock wave to the centre. Of course, if $t_{L}<t_{s}$ the shell is completely ablated, which is characteristic of an exploding pusher regime and provides us with a second, more severe, criterion to characterize this regime. The dark area in figure 13 shows the cases at $I_{\mathrm{abs}}=10^{15} \mathrm{~W} \mathrm{~cm}^{-2}$ for which the laser breaks through the shell at time $t_{L}<t_{s}$, whereas for the cases at $I_{\mathrm{abs}}=10^{14} \mathrm{~W} \mathrm{~cm}^{-2}$ the laser never penetrates the fuel. It can be seen that the condition $t_{L}<t_{s}$ is more restrictive that the condition $\rho_{\max }<\rho_{0}$; indeed, there are exploding pusher cases for which the laser does not penetrate the fuel, nevertheless the shell ablation avoids the formation of a confining shell $\left(\rho_{\max }<\rho_{0}\right)$.

Figure 14 concerns hydrodynamics of the implosion such as the absorbed energy $\left(E_{\mathrm{abs}}\right)$, the implosion velocities $(V)$ and the CRs, whereas Figure 15 presents the bang time $\left(B_{t}\right)$, the time of the arrival of the first shock to the capsule centre $\left(t_{s}\right)$ and their difference $B_{t}-t_{s}$. As the calculations use fixed laser absorbed intensities $I_{\text {abs }}\left(\mathrm{W} \mathrm{cm}^{-2}\right)$, the absorbed energy grows with the capsule radius $r$ and the shell thickness. The absorbed laser profile grows linearly in $300 \mathrm{ps}$, remains constant until $t_{s}$ and decreases to zero in $300 \mathrm{ps}$; thus, assuming 

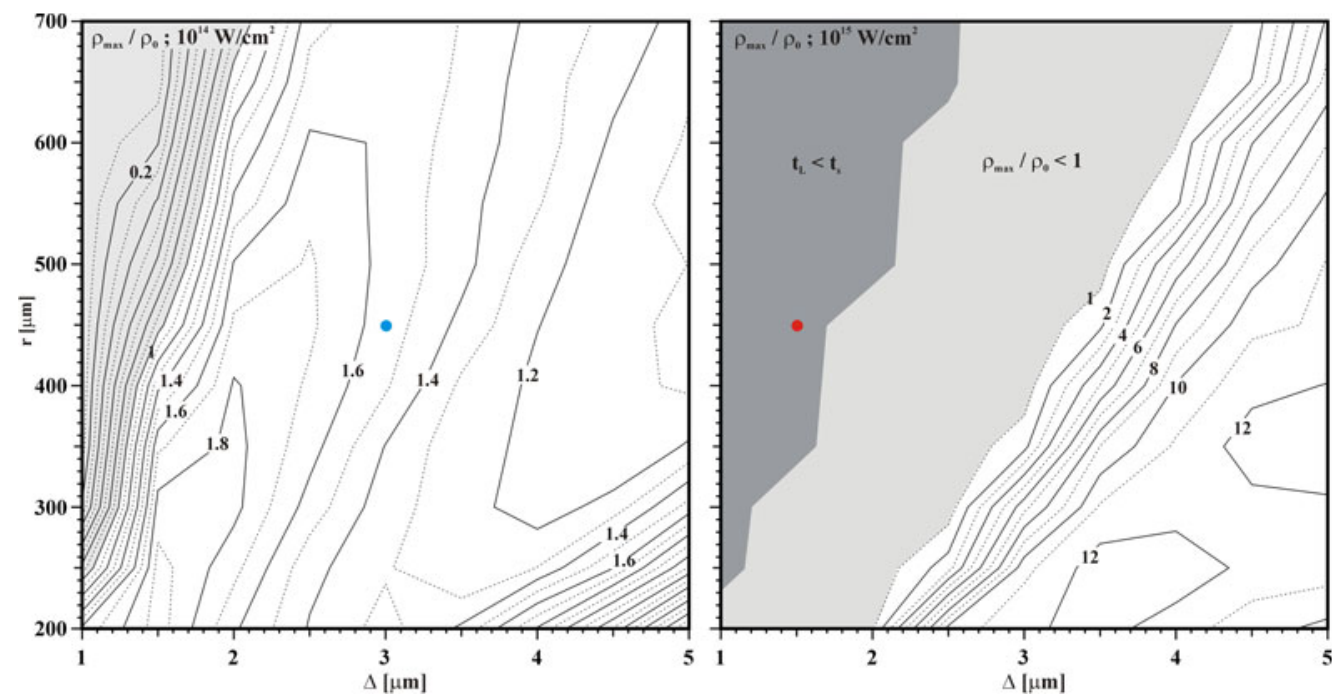

FIGURE 13. Contour maps of the ratio $\rho_{\max } / \rho_{0}$ evaluated at the bang time as a function of the capsule radius $r$ and shell thickness $\Delta$. Lightly shaded areas enlighten the cases for which $\rho_{\max } / \rho_{0}<1$, whereas the dark areas correspond to the cases where also $t_{L}<t_{s}$.

that $t_{s}>300 \mathrm{ps}$, the laser absorbed energy $\left(E_{\mathrm{abs}}\right)$ grows with the square of the target radius $\left(\propto r^{2}\right)$ and the time $\left(\propto t_{s}\right): E_{\mathrm{abs}}=4 \pi r^{2} I_{\mathrm{abs}} t_{s}=0.125[\mathrm{~kJ}](r / 100 \mu \mathrm{m})^{2}\left(I_{\mathrm{abs}} / 10^{14}\right)\left(t_{s} / 1 \mathrm{~ns}\right)$. For capsules of some hundred micrometres in radius, the absorbed energy is of a few kilojoules for $I_{\mathrm{abs}}=10^{14} \mathrm{~W} \mathrm{~cm}{ }^{-2}$ and 10 times greater when $I_{\mathrm{abs}}=10^{15} \mathrm{~W} \mathrm{~cm}^{-2}$. The current specifications (CEA/DAM 2020) of the LMJ facility allow shaped laser pulses with a duration from 0.7 to $25 \mathrm{~ns}$ with a maximum total energy of $1.3 \mathrm{MJ}$ and a maximum power of $400 \mathrm{TW}$ on the target.

Concerning $\mathrm{CR}=r / r_{\min }$ (where $r$ is the initial capsule radius and $r_{\min }$ is the minimum radius of the compressed fuel), the massive pusher regime is characterized by a CR greater than 5 and approaching 20. Thus, any illumination non-uniformity greater than $10 \%$ would imply a shell deformation greater than $100 \%$ at stagnation that can lead to a breakdown of the shell. Such a regime is less robust than the exploding pusher regime that concerns a CR below 5 and consequently the implosion supports higher non-uniformities. We can see also that for both intensities, it is possible to achieve both regimes, depending on the shell thickness and target radius. The frontier between these regimes indicates that the two parameters (thickness and radius) as related as follows: the higher the radius, the thicker the shell. For LMJ, the search for an exploding pusher regime should prompt consideration of the dark or grey zone.

All these calculations cover a huge range of implosion velocities $V$, from 150 to $750 \mu \mathrm{m}$ $\mathrm{ns}^{-1}$. This has strong implications on the level of acceleration and of the hydrodynamic stability of the capsule. Comparing the variation of the velocity with the variation of the bang time $B_{t}$, it is worth noting that the direction of the velocity gradient in the $(r, \Delta)$ plane is roughly perpendicular to the gradient of the bang time in the massive pusher regime whereas both gradients are almost aligned in the exploding pusher regime. Assuming that ablation velocity is constant for a given intensity, $v \sim g t$ and $t \propto B_{t}$, the amplification of the Rayleigh-Taylor instability (RTI) can be estimated as $A / A_{0} \sim \exp \left(\sqrt{k v t}-k v_{a} t\right)$ (Bodner 1974; Takabe et al. 1985). 

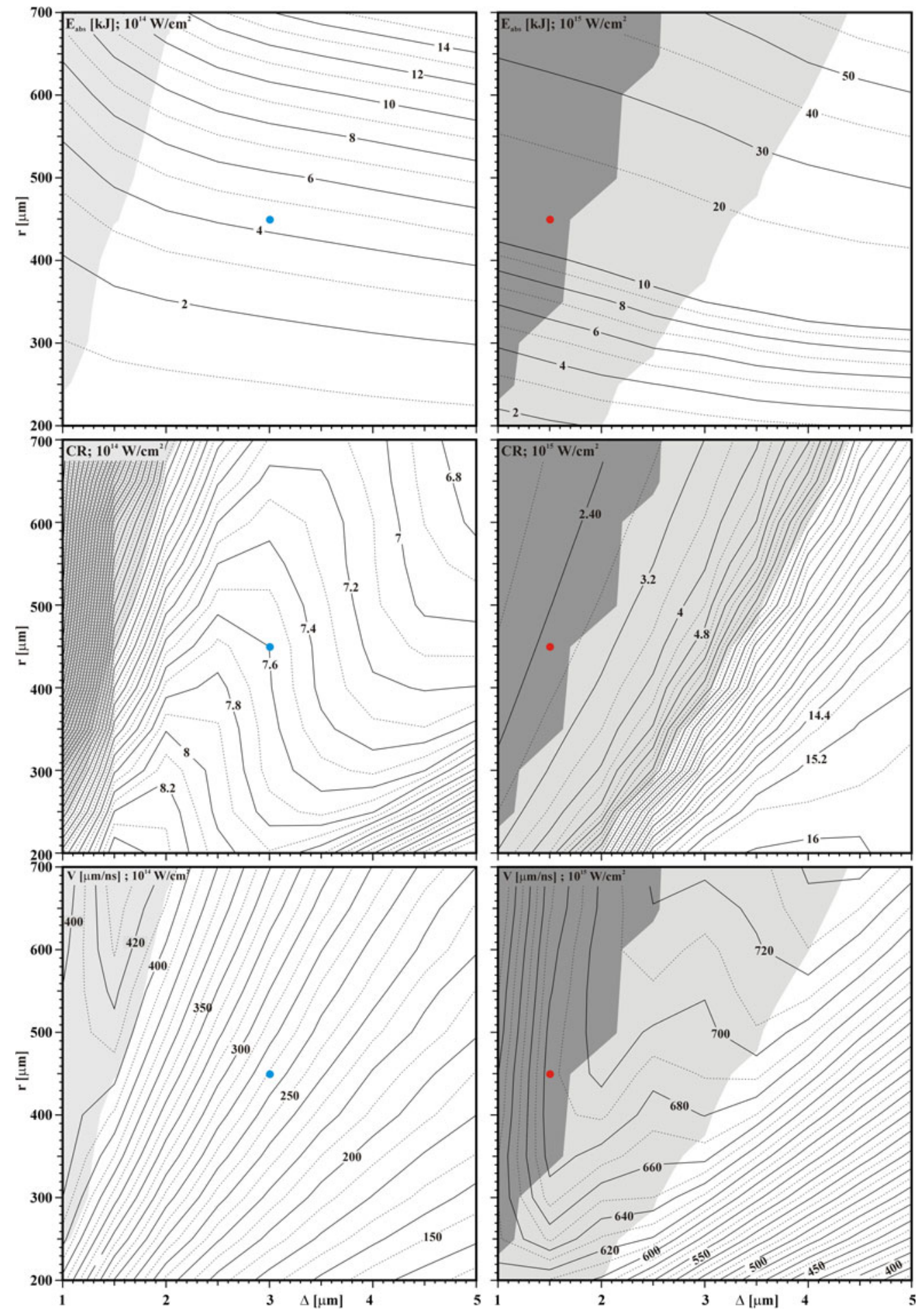

FIGURE 14. Absorbed energy $E_{\mathrm{abs}}, \mathrm{CR}$ and implosion velocity $V$. 

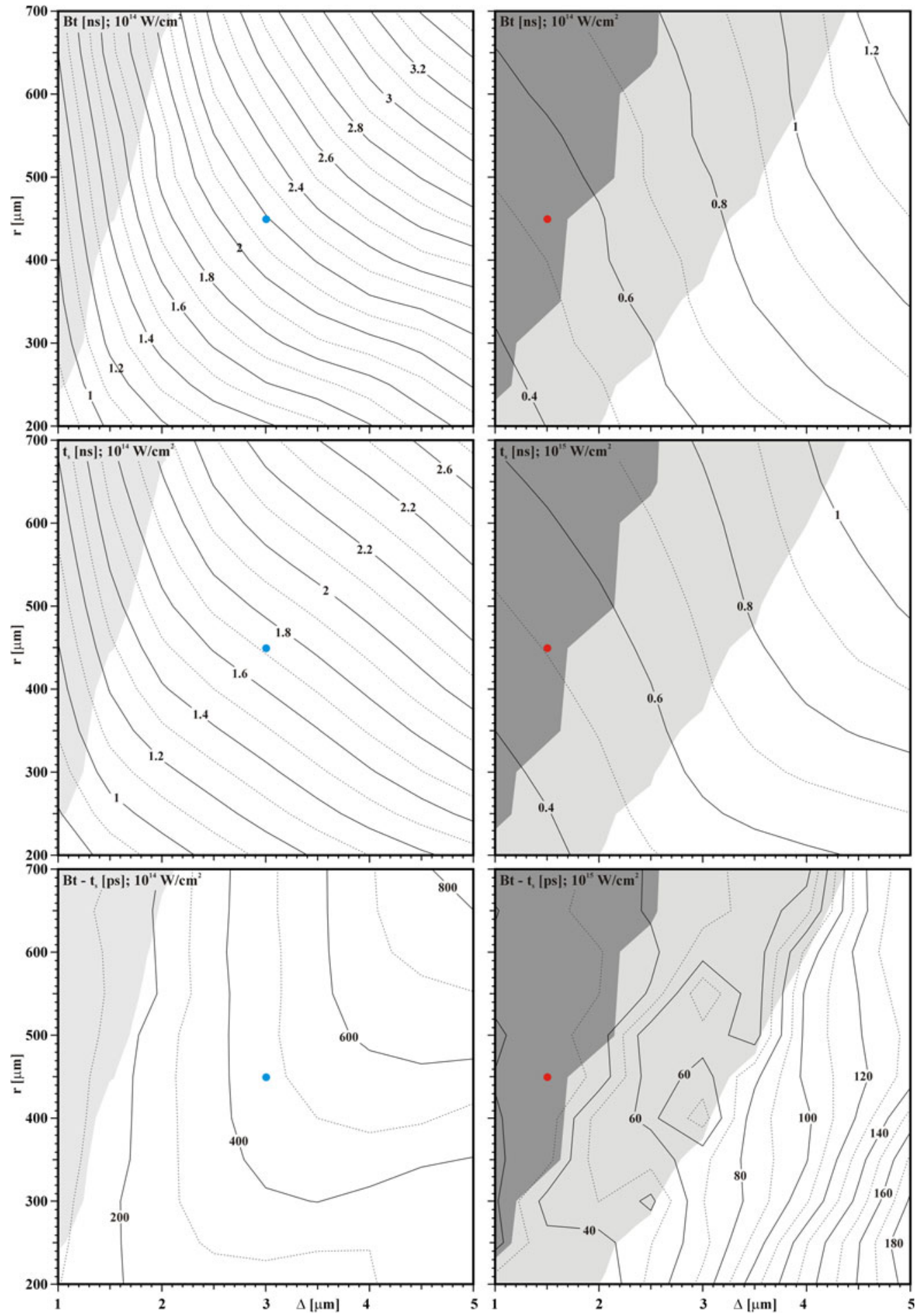

FIGURE 15. Bang time $B_{t}$, arrival of the first shock wave to the centre $t_{s}$, and the difference $B_{t}-t_{s}$. 
When looking at figures 14 and 15, it is worth noting that implosions would not have the same sensitivity to RTI depending on the regions in the parameter space. In fact, we can argue that a large radius and thick shell under high intensity would be less robust to RTI than an exploding pusher regime. Indeed, bang time $B_{t}$ defined as the time of maximum fusion power grows with the capsule radius and is considerably shorter for the cases of exploding pusher in comparison with the corresponding massive pusher. The former should leave less time for RTI to grow.

Another important parameter is the time of the arrival of the first shock wave to the capsule centre, $t_{s}$. As for the bang time, $t_{s}$ grows with the fuel radius and is considerably larger for the cases working at $I_{\mathrm{abs}}=10^{14} \mathrm{~W} \mathrm{~cm}^{-2}$ in comparison with the cases of $I_{\mathrm{abs}}=10^{15} \mathrm{~W} \mathrm{~cm}^{-2}$. Differences between the bang time and the time of the arrival of the first shock wave $B_{t}-t_{s}$ are also plotted in figure 15 . This parameter indicates how fast the hydrodynamic process is. The cases of massive pusher provide a difference of few hundred picoseconds, whereas in the exploding pusher cases the difference is reduced to tens of picoseconds. When this difference becomes shorter and shorter, the stagnation and the confinement times are very short, given by the time spent by returning shock to meet the stagnating shell. In contrast, when this time difference becomes larger, the shock has time to return towards the massive shell and reflects at the gas-pusher interface and collapses again at the target centre. This can occur many times during the end of deceleration. In that case, mainly concerning the massive pusher regime, the confinement time becomes very long (hundreds of picoseconds). This is preferentially achieved for low intensities, high radius and thick shell, when the implosion velocity is low, roughly $200 \mu \mathrm{m} \mathrm{ns}^{-1}$. For high implosion velocities, the shock returns only once.

Other observables connected to the implosion history concern the areal densities of the fuel and of the whole target. During deceleration and stagnation, as seen in figure 12, the areal density increases up to a maximum and then decreases. This maximum is plotted in figure 16, for the whole target (total areal density $\rho R\left[\mathrm{mg} \mathrm{cm}^{-3}\right]$ ) and only for the fuel $\left(\rho R_{\mathrm{DHe}}\left[\mathrm{mg} \mathrm{cm}^{-3}\right]\right)$, versus shell thickness and target radius. Also plotted is the ratio $\rho R / \rho R_{\mathrm{DHe}}$. In the parametric variations, the gas pressure and initial density $\rho_{0}$ are kept unchanged. Thus, the initial mass of gas is proportional to the cube of initial radius and is unchanged during implosion. The compression rate of the fuel can be deduced from $\left(\rho / \rho_{0}\right)_{\mathrm{DHe}}=(\mathrm{CR})^{3}$ and the maximum fuel areal density is related to the CR by $\rho r_{\mathrm{DHe}}=$ $\rho_{0} r_{0} C R^{2}$. As these data interplay, their variations are not obvious. In particular, the areal density ratio can give an estimate of the relative importance of the fuel on the whole areal density. For instance, Frenje et al. (2019) claim that their targets were chosen to minimize the shell areal density contribution in the energy loss of charged particles coming from $\mathrm{D}^{3} \mathrm{He}$ implosions. Such a consideration should also be deduced from our numerical results.

\subsection{Sources of particles}

Complementary to these hydrodynamics considerations, numbers of particles emitted by the target are estimated in the parameter space. Figure 17 represents the total number of neutrons $n_{n}$ from the $\mathrm{D}(\mathrm{D}, n)^{3} \mathrm{He}$ reaction, the total number $n_{\alpha}$ of $\alpha$-particles [ $\mathrm{D}\left({ }^{3} \mathrm{He}, p\right) \alpha$ ] and the total number $n_{p}$ of the $14 \mathrm{MeV}$ protons $\left[\mathrm{D}\left({ }^{3} \mathrm{He}, p\right) \alpha\right]$.

The others particles are represented in figure 18, such as the total number $n_{3 \mathrm{He}}$ of ${ }^{3} \mathrm{He}$ ions $\left[\mathrm{D}(\mathrm{D}, n)^{3} \mathrm{He}\right]$, the total number $n_{T}$ of tritium $[\mathrm{D}(\mathrm{D}, p) \mathrm{T}]$ and the total number $n_{p}$ of the $3 \mathrm{MeV}$ protons $[\mathrm{D}(\mathrm{D}, p) \mathrm{T}]$. All these numbers concern the particles escaping the target, not the source term. In complement, in figure 19, we focus on the number of neutrons $n_{s}$ generated before the arrival of the first shock wave to the capsule centre $\left(t<t_{s}\right)$, and to the ratio $n / n_{s}$, where $n$ is the total number of neutrons. This allows discriminating between implosions that are more or less sensitive to low-mode asymmetries. 

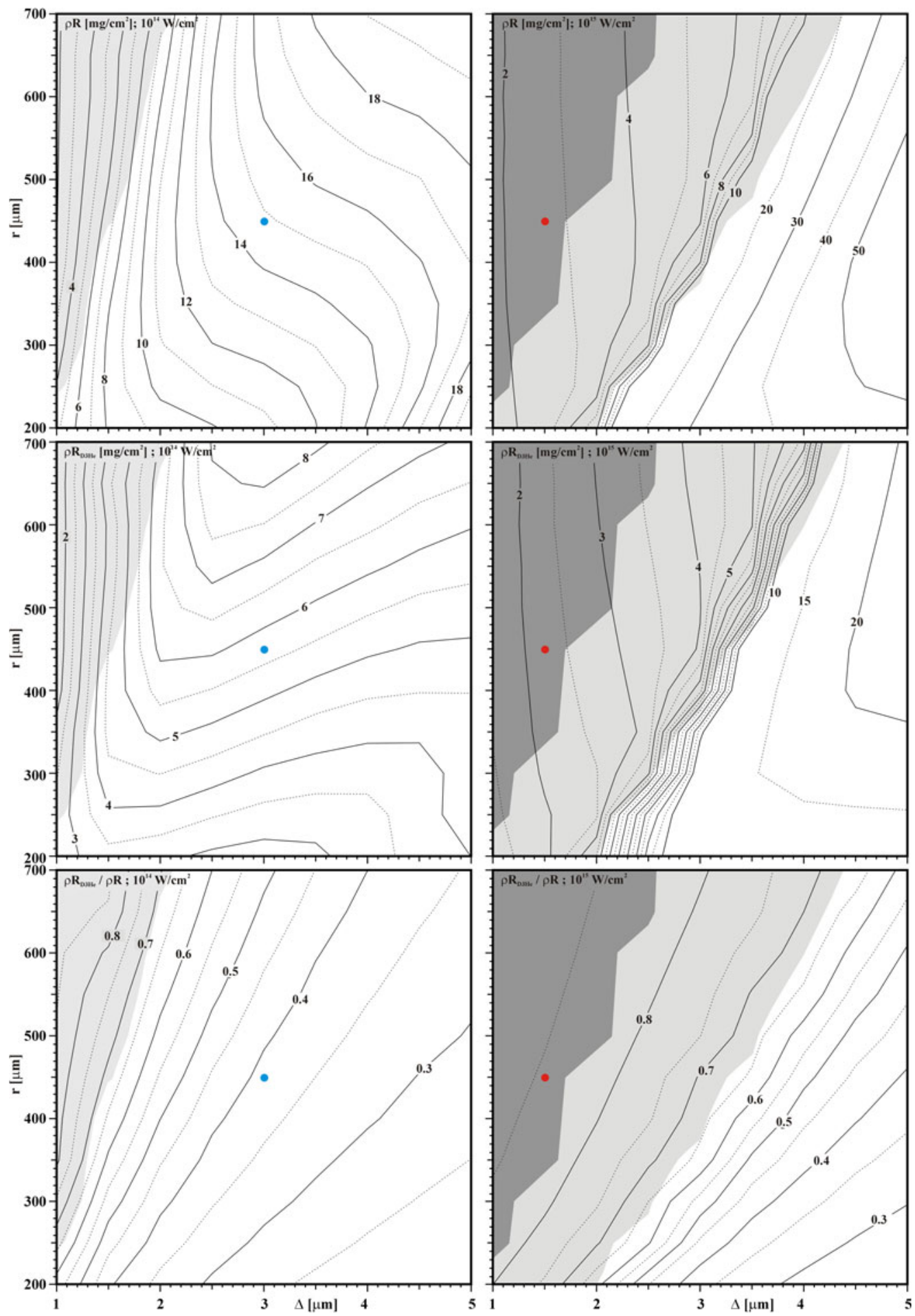

FIGURE 16. Total areal density $\rho R$, areal density of the fuel $\rho R_{\mathrm{DHe}}$ and the ratio $\rho R_{\mathrm{DHe}} / \rho R$. 
Direct-drive target designs as energetic particle sources
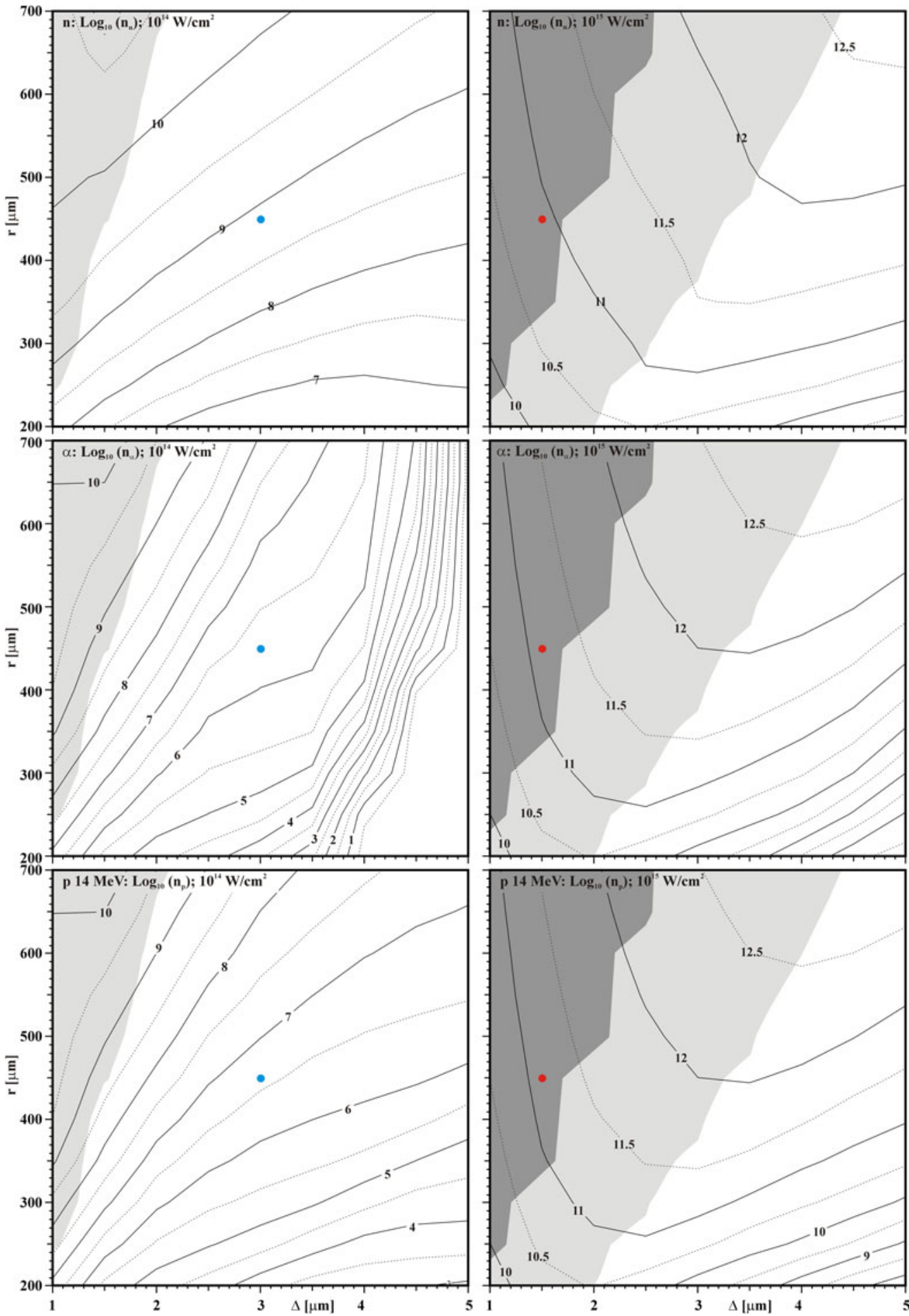

FIGURE 17. Total number of neutrons $n, \alpha$-particles and $14 \mathrm{MeV}$ protons. 

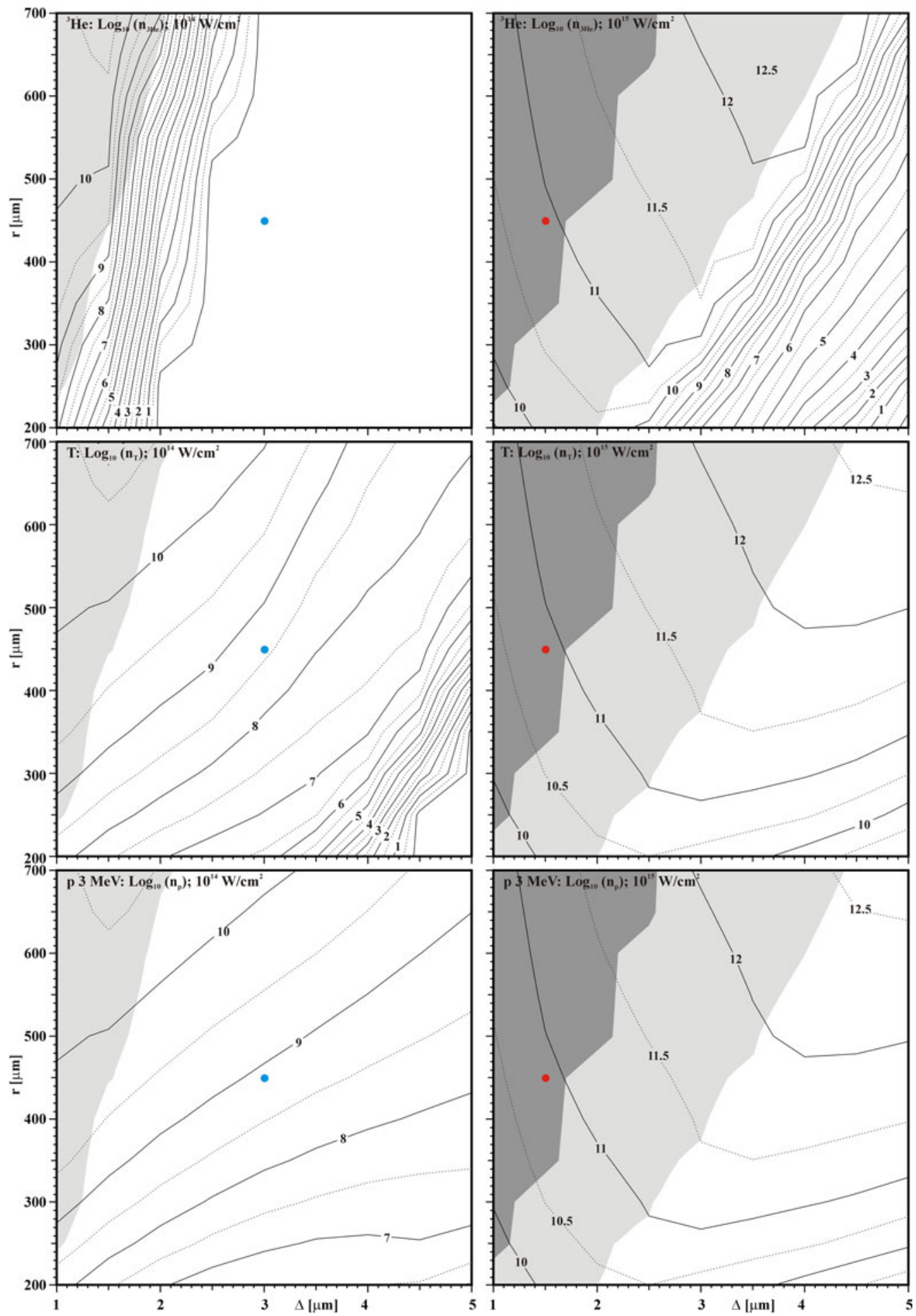

FIGURE 18. Total number of ${ }^{3} \mathrm{He}$ ions, tritium ions and $3 \mathrm{MeV}$ protons. 

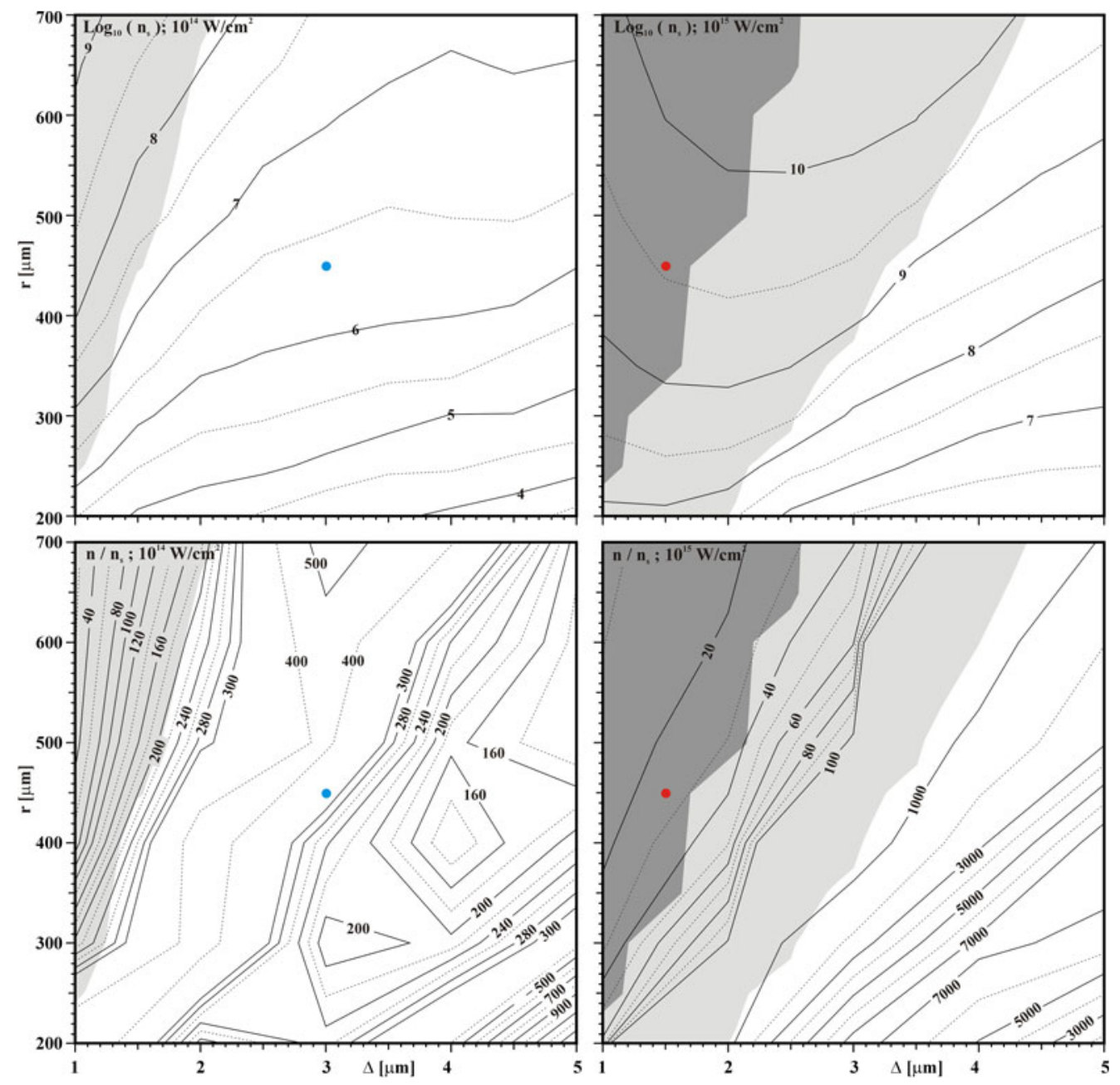

FIGURE 19. Neutrons $n_{s}$ produced before the arrival of the first shock wave to the capsule centre and neutron ratio $n / n_{s}$.

Indeed, it is possible to increase significantly the contribution of shock-produced neutrons $\left(n_{s}\right)$ to the total number of neutrons $(n)$. Considering these neutrons is very stringent. Usually, first shock-generated neutrons are those that are created during the rebound after the first shock collapse and before it encounters the gas-shell interface. Indeed, after the rebound, low-mode asymmetries could be dominant and could lead to a decrease of the neutron number due to the first shock. The shock mistiming added to shell break-up and shock distortion could make the shock focalization less efficient in terms of pressure and temperature that would be deleterious and could reduce significantly the neutron number. Considering $n_{s}$ is much more conservative in that before the first shock collapse, the neutrons created are not influenced by the low-mode asymmetries of the incoming shock and depend directly on the pressure in the post-shock medium. The strength of the shock is thus not altered by these asymmetries and depends directly on the ablation pressure created by the laser pulse and also on the impedance adaptation at the gas-shell interface. In addition, shell break-up is not a concern. On the other hand, the contribution of these 

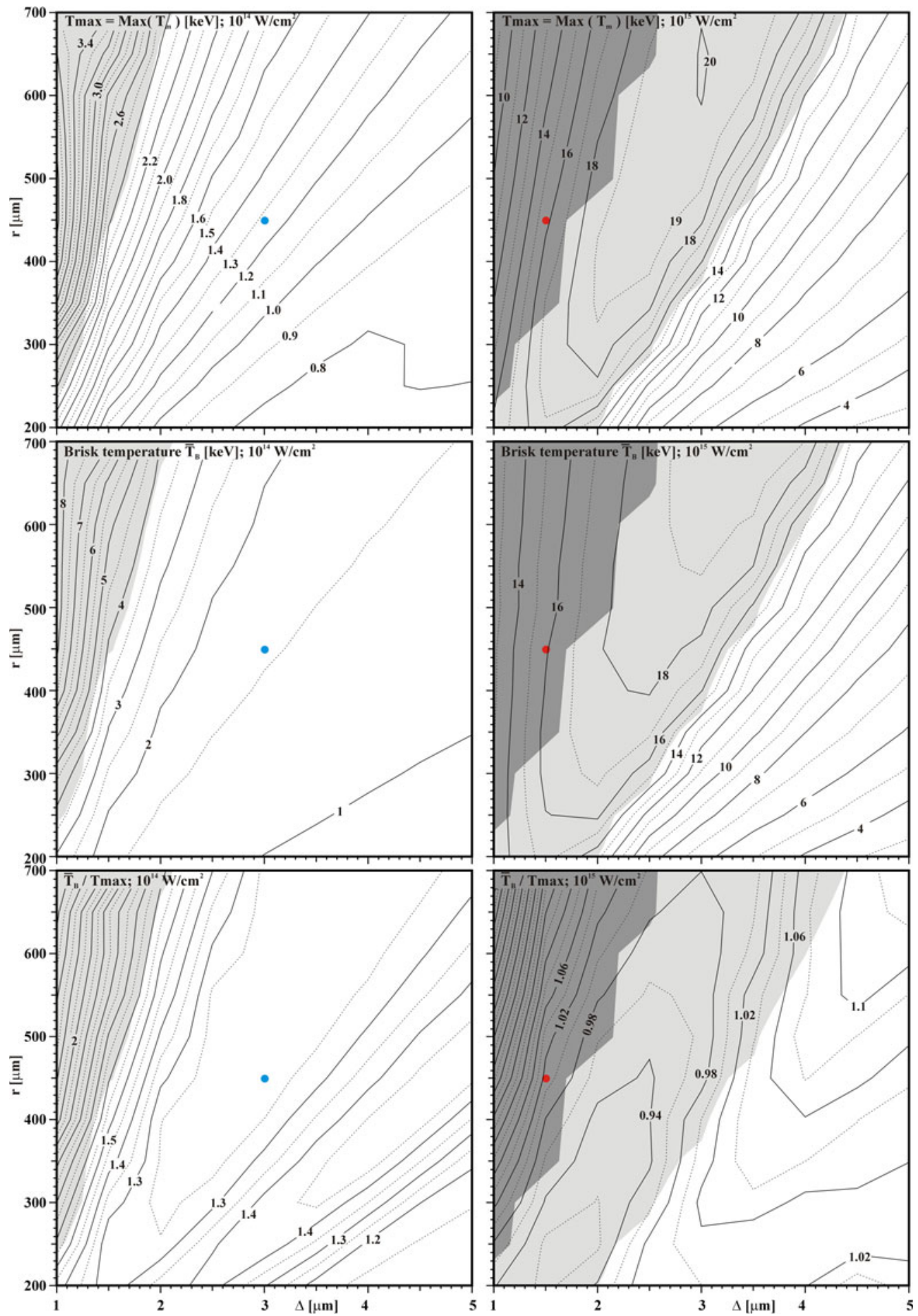

FIGURE 20. Maximum mass-average fuel temperature $T_{\max }$, Brysk temperature $T_{B}$ and temperature ratio $T_{B} / T_{\max }$. 
neutrons to the whole number is small as can be seen in figure 19 and never exceeds $5 \%$ of the total number of neutrons. Despite this, it appears possible to produce more than $10^{10}$ neutrons when laser intensity is $10^{15} \mathrm{~W} \mathrm{~cm}^{-2}$.

\subsection{Neutron-related temperatures and areal density measurements}

As explained previously, it is possible to infer temperature and areal density by using the particle information escaping the target. The simulations of the nuclear diagnostics that give such information are compared with the data directly extracted from the simulations. Figure 20 gives the maximum mass-averaged temperature $T_{\max }=\operatorname{Max}\left[T_{m}\right]$ reached in the fuel, the Brysk's temperature $\bar{T}_{B}$ provided by the time-integrated neutron spectra and the temperature ratio $\bar{T}_{B} / T_{\max }$. As in $\S 3$, both temperatures match quite well in the exploding pusher regime and the ratio is close to unity in the major part of the parametric space, whereas in the massive pusher regime, significant differences persist in the entire parametric space. This must drive our attention to use these nuclear measurements carefully.

We also represent, in figure 21, the ratio $(\rho R)_{M} /(\rho R)_{1}$, where $(\rho R)_{M}$ is the maximum areal density provided by the hydro-calculation and $(\rho R)_{1}$ is the maximum areal density estimated using the energy loss of the $14 \mathrm{MeV}$ protons and $(\rho R)_{M} /(\rho R)_{2}$, where $(\rho R)_{2}$ is the maximum areal density estimated using the time-integrated average energy loss of the $14 \mathrm{MeV}$ protons (see $\S 4$ and figure 12).

It is found that MULTI areal density is not systematically well reproduced by the maximum of the time-resolved energy losses of the $14 \mathrm{MeV}$-protons. More restricting measurement should have to be considered; however, it is worth noting that for the massive pusher regime $(\rho R)_{M} /(\rho R)_{1}$ is within $10 \%$ in large part of the parametric space.

\subsection{Particle spectra}

In order to analyse the variations of the spectra of escaping particles in the parameter space, the data are grouped by laser intensity. First considered is $I_{\mathrm{abs}}=10^{14} \mathrm{~W} \mathrm{~cm}^{-2}$. In figures 22 and 23, the contour maps of the left column show the differences $\Delta \epsilon$ between the average energy of the generated particles $\varepsilon=\varepsilon_{0}+B(T)$ (see (3.3)) and the average energy of the time-integrated particle spectra $E_{a}: E_{a}^{i}=\int n_{i}(\varepsilon) \mathrm{d} \varepsilon / N_{i}$ (see $\left.\S 3\right), \Delta \varepsilon=\langle\varepsilon\rangle-$ $E_{a}$. This difference is equivalent to the average energy loss of the particles as described in Frenje et al. (2019). It considers the whole plasma contributing to the losses, i.e. the fuel and the shell. All the particles are considered here. In the right column, the calculations consider only the energy losses in the fuel. In this case, the difference $\Delta \varepsilon^{*}=\langle\varepsilon\rangle-E_{a}^{*}$, is obtained by subtracting the energy $E_{a}^{*}$ that has been calculated neglecting the particle energy loss in the $\mathrm{SiO}_{2}$ material. For some peculiar parameters, particles cannot escape (in particular, the heavy and less energetic ${ }^{3} \mathrm{He}$ and $\mathrm{T}$ ) and are trapped in the target, and more specifically in the shell. This mainly happens when the $\mathrm{SiO}_{2}$ shell is only marginally ablated (small capsule radius $r$ and large $\Delta$, see $\rho R_{\mathrm{DHe}} / \rho R$ in figure 16). This suggest that if some areas of the shell are more ablated than others, e.g. due to non-uniform irradiation, the ${ }^{3} \mathrm{He}$ and $\mathrm{T}$ particles would preferably escape from surfaces with smaller areal density. Therefore, the spectra of the particles evaluated at different angles may reflect in some way the non-uniformity of the irradiation.

It is worth noting that, at this intensity, it is very difficult to maximize the energy loss in the fuel compared with the loss in the shell. Indeed, the shell contribution to the energy loss is always greater than $50 \%$ for all particles. This is consistent with the fuel areal density that is never dominant compared with the shell areal density.

Analogous analysis is given in figures 24 and 25 for the absorbed intensity of $10^{15} \mathrm{~W}$ $\mathrm{cm}^{-2}$. Conversely to the lower intensity, it is possible to find a region in the parametric 

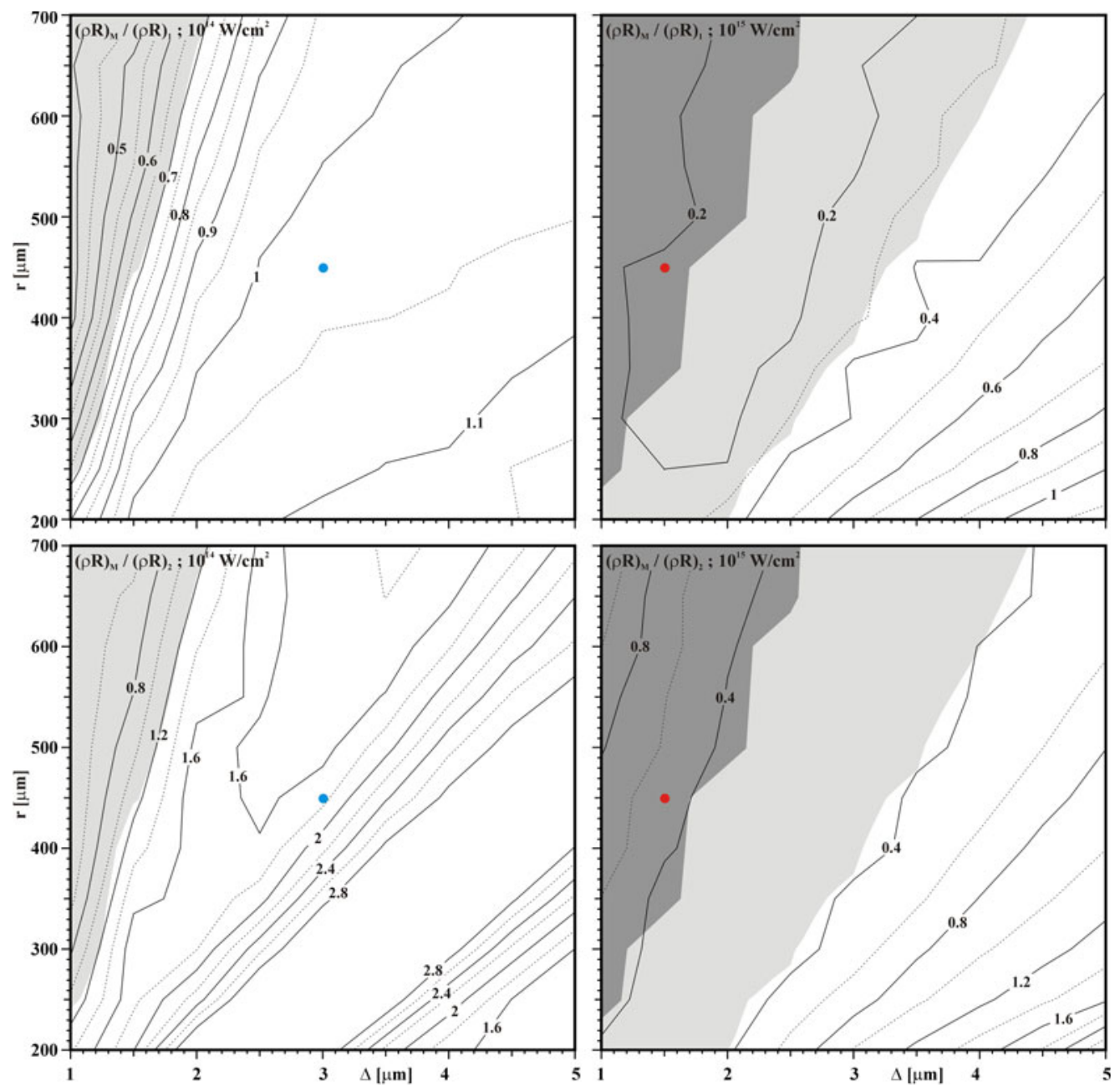

FIgURE 21. Ratio $(\rho R)_{M} /(\rho R)_{1}$ and $(\rho R)_{M} /(\rho R)_{2}$, with $(\rho R)_{M},(\rho R)_{1}$ and $(\rho R)_{2}$ the areal density provided by MULTI, the $14 \mathrm{Me}$-proton time resolved and averaged spectra, respectively.

space where the energy loss is dominant in the fuel. In that case, the problem of remaining shell is removed. However, the energy loss is very weak, around $20 \mathrm{keV}$ that could be below the resolution of particle spectrometers. For applications as a particle source the interest would be in minimizing the total energy loss through the capsule, whereas the energy lost should be accurately tuned if the intention is to use the particles as a diagnostic of the implosion.

To conclude this part, the tendency to reduce the contribution of the shell in the energy loss of energetic particle is not obvious and represents a difficult to achieve trade-off.

Another observable is the standard deviation $(\sigma)$ of the time-integrated spectrum of the particle escaping the target. This is represented in figure 26 (figure 27) for all particles, $\alpha$, $14 \mathrm{MeV}$ protons, ${ }^{3} \mathrm{He}$, neutrons, tritium and $3 \mathrm{MeV}$ protons, for the cases at the absorbed intensities of $10^{14} \mathrm{~W} \mathrm{~cm}^{-2}\left(10^{15} \mathrm{~W} \mathrm{~cm}^{-2}\right)$. As mentioned previously in $\S 3.1, \sigma$ depends on plasma temperature and on the stopping power energy loss. Larger energy loss implies larger $\sigma$, but larger $\sigma$ does not necessarily imply larger energy loss as it could also result 

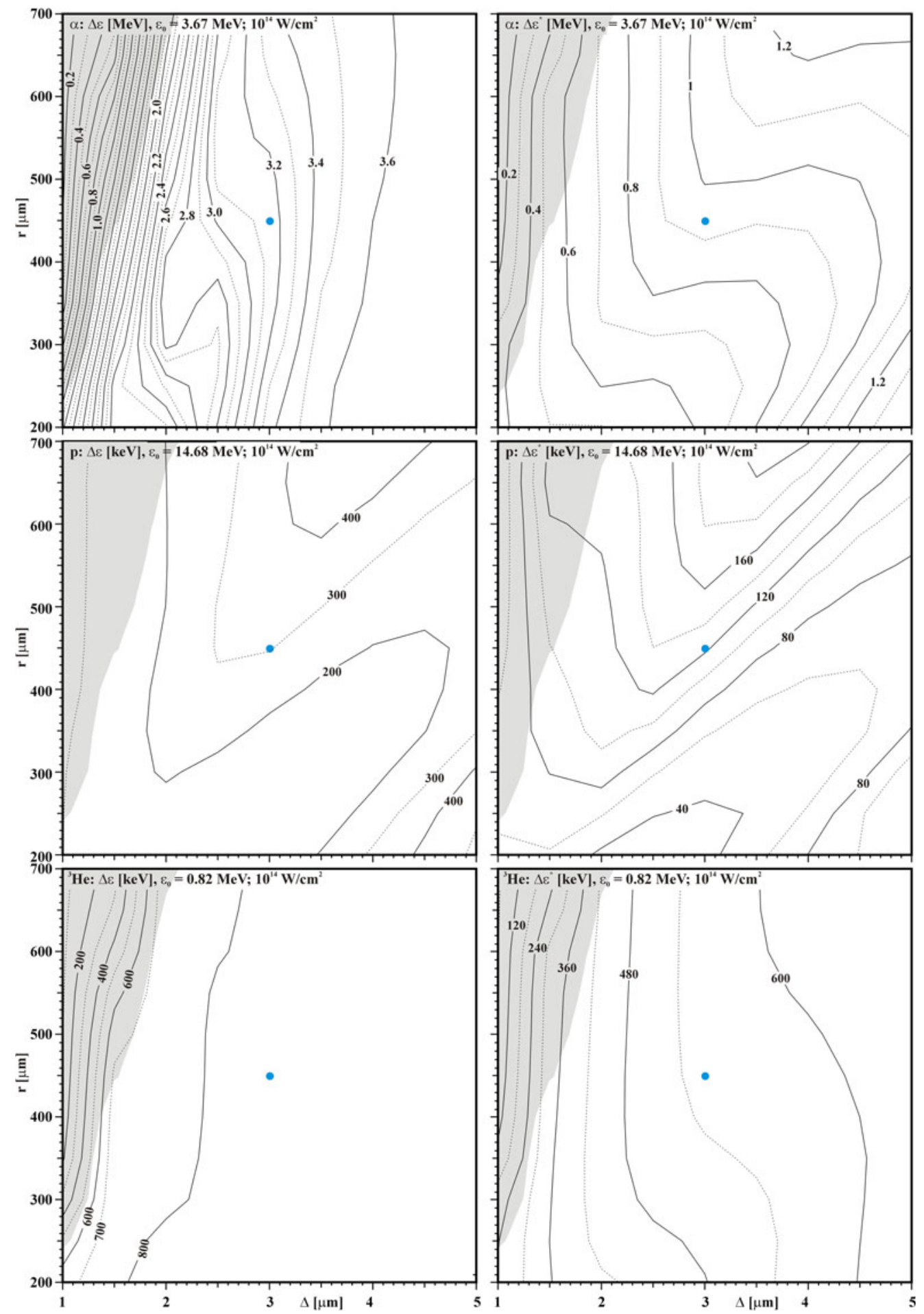

FIGURE 22. Energy loss $\Delta \epsilon$ of $\alpha, 14-\mathrm{MeV}$ protons and ${ }^{3} \mathrm{He}$ ions in the whole plasma (left column) and in the fuel only (right column). Here $I_{\mathrm{abs}}=10^{14} \mathrm{~W} \mathrm{~cm}^{-2}$. 

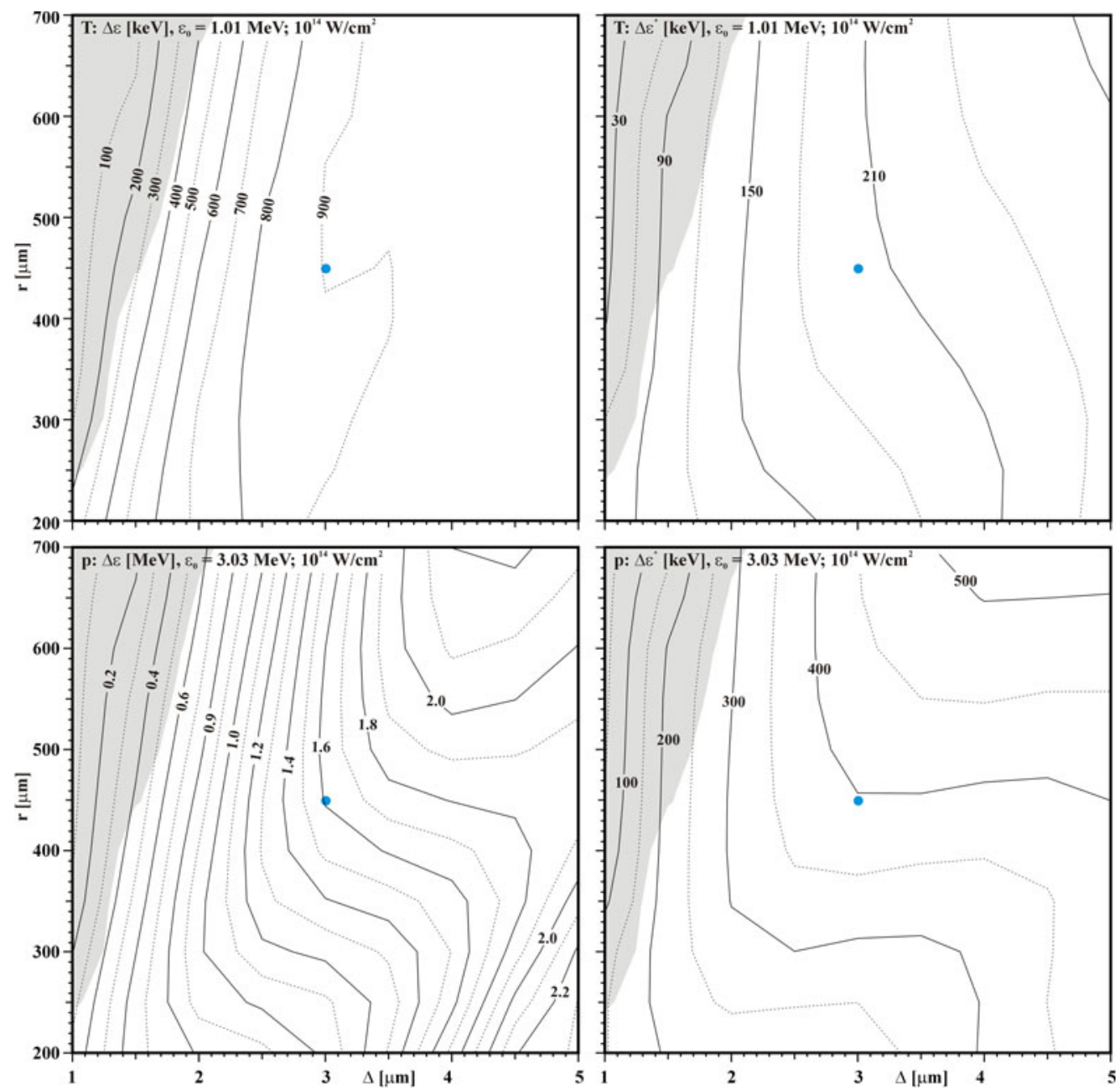

FIGURE 23. Energy loss $\Delta \epsilon$ of $T$ and 3-MeV protons in the whole plasma (left column) and in the fuel only (right column). Here $I_{\mathrm{abs}}=10^{14} \mathrm{~W} \mathrm{~cm}^{-2}$.

from higher temperatures. When $\sigma$ becomes larger than the average output energy of the particle $\left(E_{a}\right)$, the energy loss is very important. This could give a measure of the particle trapping at a certain time during the particle emission. To conclude this part, it is worth noting that the energy of the output particles varies significantly with the shell thickness. Thus, the thickness can be chosen to tune the energy of the source. For specific applications, this could be fruitful.

\section{Conclusions}

In this paper, we have presented a large study of one-dimensional numerical simulations of the implosions of $\mathrm{D}^{3} \mathrm{He}$ gas-filled $\mathrm{SiO}_{2}$ glass capsules. Two distinct regimes have been identified: an exploding pusher regimes and a massive pusher regime. The first regime is characterized by a strong converging shock and a small residual mass of the shell at stagnation, whereas in the second case a massive stagnating shell compresses the central gas at high temperatures. In the first regime, a thin glass $\left(\mathrm{SiO}_{2}\right)$ spherical layer is lightened 

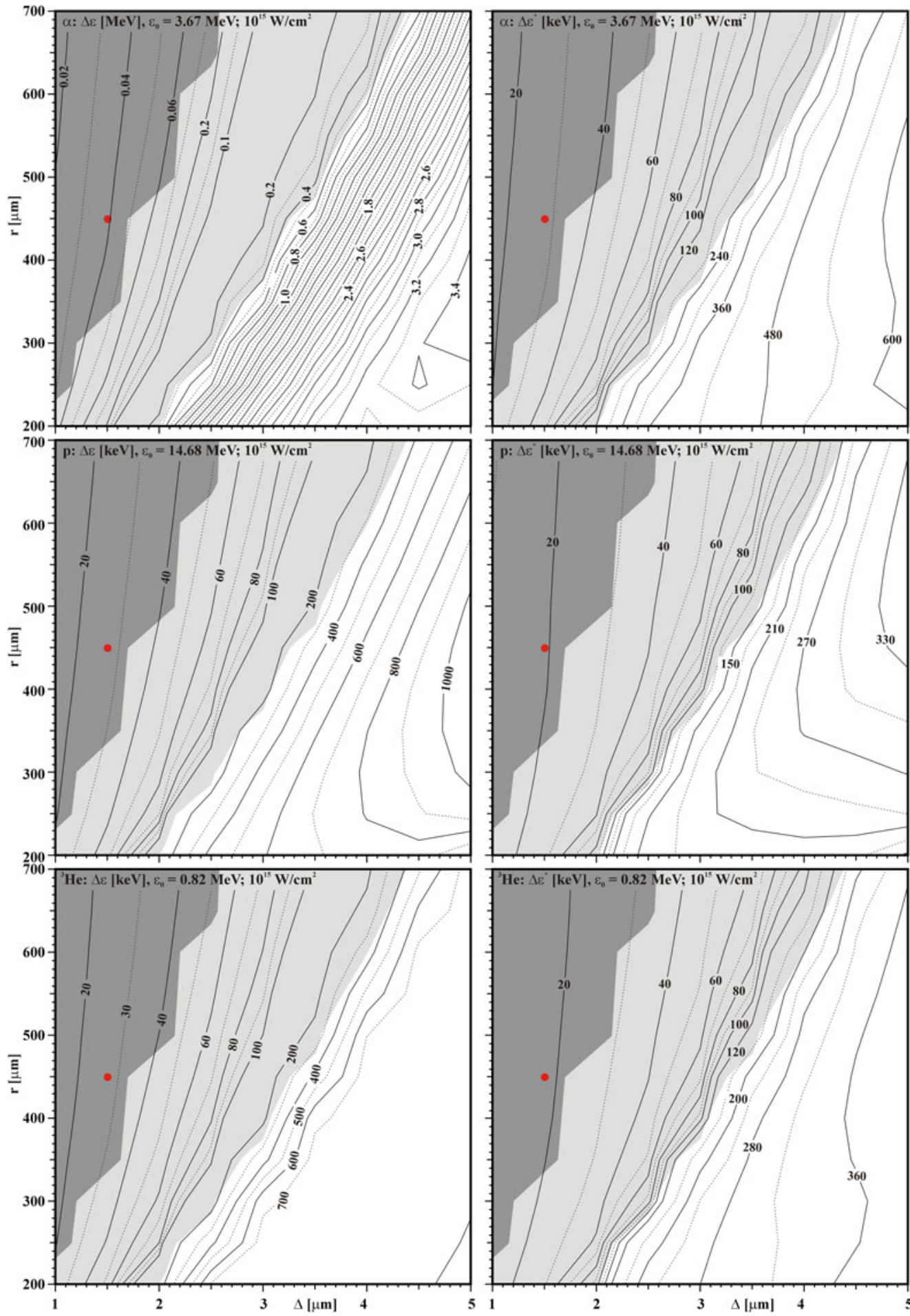

FIGURE 24. Energy loss $\Delta \epsilon$ of $\alpha, 14-\mathrm{MeV}$ protons and ${ }^{3} \mathrm{He}$ ions in the whole plasma (left column) and in the fuel only (right column). Here $I_{\mathrm{abs}}=10^{15} \mathrm{~W} \mathrm{~cm}^{-2}$. 

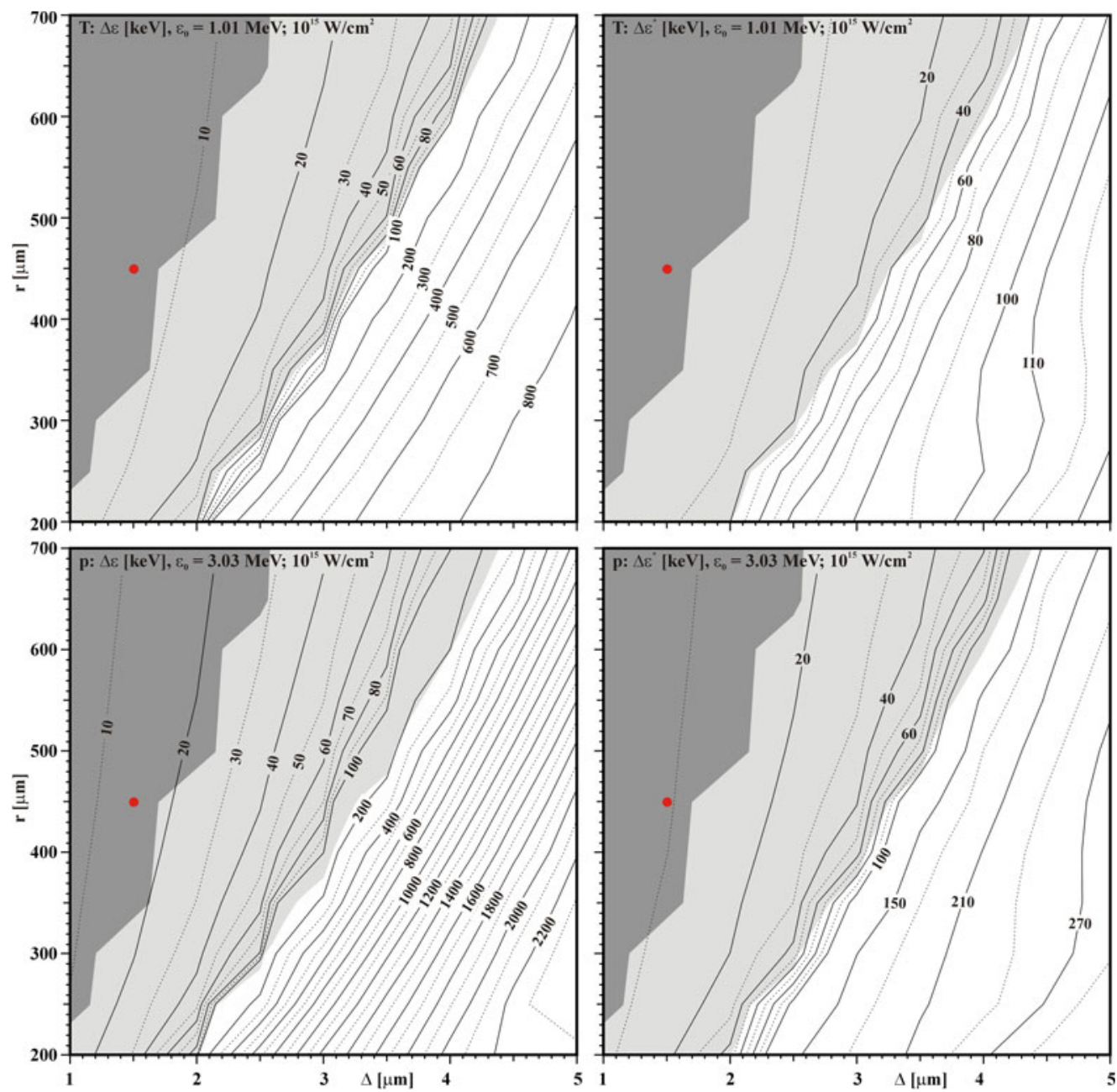

FIGURE 25. Energy loss $\Delta \epsilon$ of $\mathrm{T}$ and $3-\mathrm{MeV}$ protons in the whole plasma (left column) and in the fuel only (right column). Here $I_{\mathrm{abs}}=10^{15} \mathrm{~W} \mathrm{~cm}^{-2}$.

by a high laser-absorbed intensity of $10^{15} \mathrm{~W} \mathrm{~cm}^{-2}$, whereas for the massive pusher regime, a low laser intensity of $10^{14} \mathrm{~W} \mathrm{~cm}^{-2}$ is absorbed in a thick glass shell. The differences between both regimes are analysed in terms of neutrons and charged particle numbers, ionic temperatures, areal densities and implosion histories. One of the main differences concerns the number of neutrons $\left(n_{s}\right)$ produced before the collapse of the first shock. In an exploding pusher regime, this number is significantly higher than for the massive pusher regime for which it stays at a low level compared with the total number of neutrons. This particularity makes this regime more robust to anisotropies of implosion, especially regarding low-mode asymmetries. Indeed, at the limit, a bipolar irradiation should produce the same level of neutrons $n_{s}$ as a purely spherical irradiation. This is why such an exploding pusher regime is specifically interesting for LMJ as an additional source of particles to serve as diagnostic in dedicated experiments. A part of the LMJ, beams could be employed to create this source. In order to offer to experimentalists a global view of possibilities for creating particle sources, we have presented a parametric variation on 

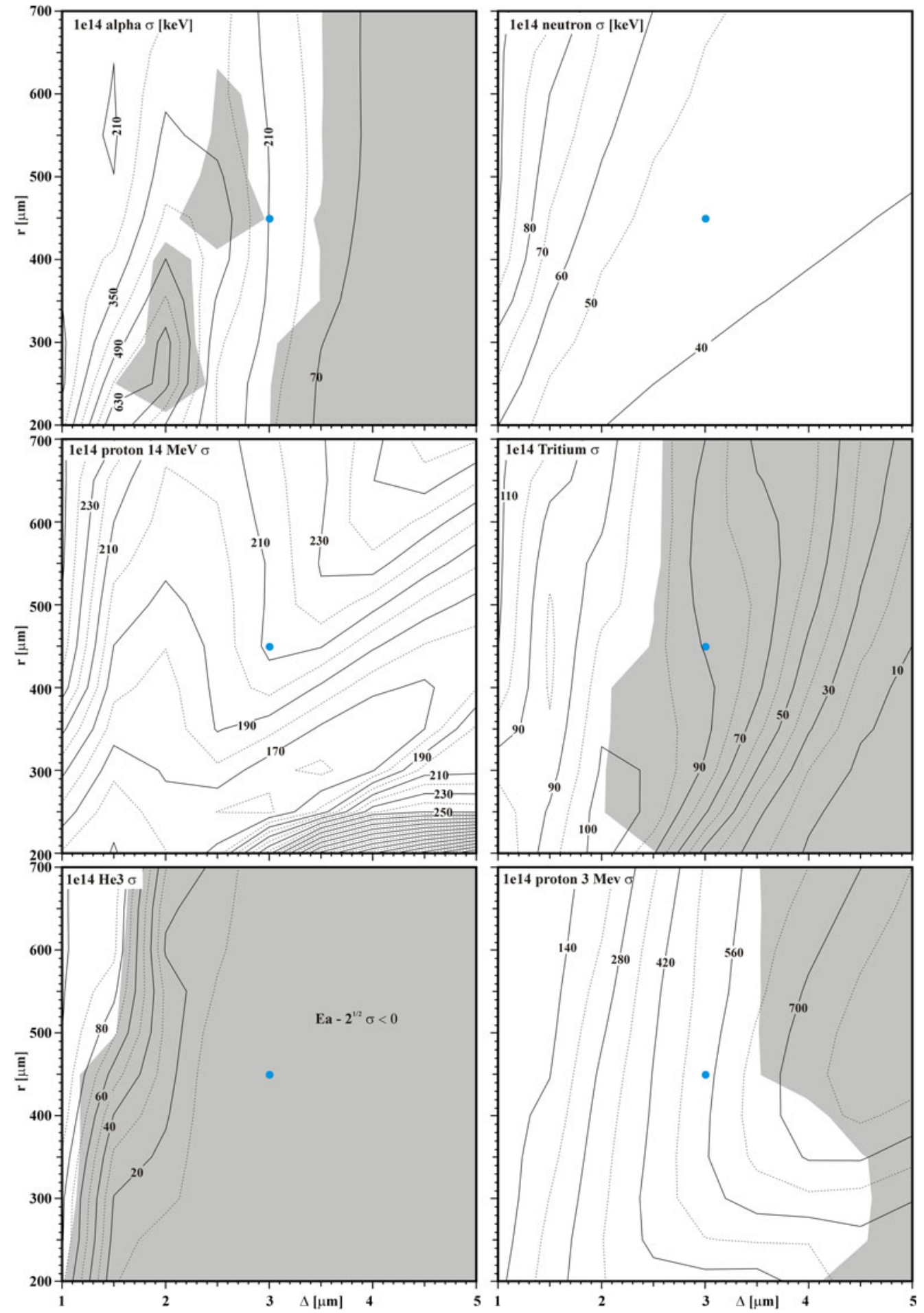

FIGURE 26. Standard deviation $\sigma$ of particle spectra for $I_{\mathrm{abs}}=10^{14} \mathrm{~W} \mathrm{~cm}^{-2}$. Grey part represents the area where the difference $\sigma-E_{a}<0$. 

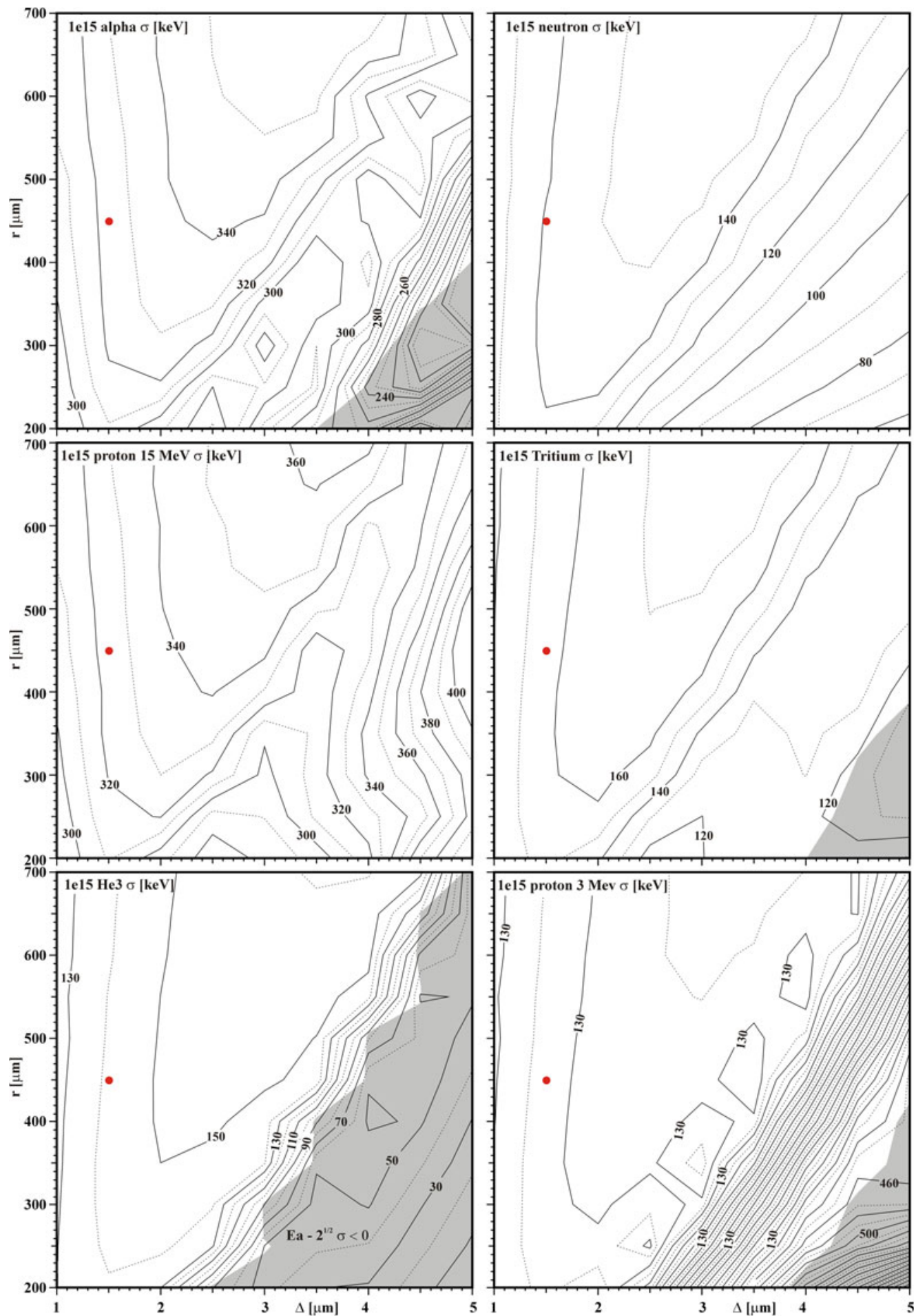

FIGURE 27. Standard deviation $\sigma$ of particle spectra for $I_{\mathrm{abs}}=10^{15} \mathrm{~W} \mathrm{~cm}^{-2}$. Grey part represents the area where the difference $\sigma-E_{a}<0$. 
the shell thickness and the inner target radius for both intensities. This study spans the parametric space and gives the opportunity to choose a regime adapted to the desired diagnostic. The energy of the particles varies significantly with the shell thickness and the type of particle. In that sense, the study presented here has allowed tuneable-energy particle sources to be proposed.

This collection of calculations should serve as a guideline; nevertheless, dedicated numerical simulations are needed in order to design a specific experiment. However, our paper aims to propose a first open view of opportunities that can be offered by the LMJ facility and the tuneable particle sources.

\section{Acknowledgements}

The authors would like to thank the referees for the fruitful suggestions that improved the manuscript.

Editor Antoine C Bret thanks the referees for their advice in evaluating this article.

\section{Funding}

This work was supported by the CEA-ENS LRC-MESO (M.T., grant number 2018-011) and the Spanish Ministerio de Ciencia Innovacion y Universidades (R.R., grant number RTI2018-098801-B-100).

\section{Declaration of interests}

The authors report no conflict of interest.

\section{Author contributions}

All authors contributed equally to analysing data and reaching conclusions, and in writing the paper.

\section{REFERENCES}

Atzeni, S. \& Meyer-Ter-Vehn, J. 2004 The Physics of Inertial Fusion. Oxford Science Press.

BodNer, S. E. 1974 Rayleigh-Taylor instability and laser-pellet fusion. Phys. Rev. Lett. 33, 761.

Bosch, H. S. \& HALE, G. M. 1992 Improved formulas for fusion cross-sections and thermal reactivities. Nucl. Fusion 32, 611.

Brandon, V., Canaud, B., Primout, M., Laffite, S. \& Temporal, M. 2013 Marginally igniting direct-drive target designs for the Laser MegaJoule. Laser Part. Beams 31, 141.

Brandon, V., Canaud, B., Temporal, M. \& Ramis, R. 2014 Low initial aspect-ratio direct-drive target designs for shock- or self-ignition in the context of the Laser MegaJoule. Nucl. Fusion 54, 083016.

BRYsK, H. 1973 Fusion neutron energies and spectra. Plasma Phys. 15, 661.

Cayzac, W., Bagnoud, V., Basko, M. M., Blazevic, A., Frank, A., Gericke, D. O., Hallo, L., Malka, G., Ortner, A., Tauschwitz, A., et al. 2015 Predictions for the energy loss of light ions in laser-generated plasmas at low and medium velocities. Phys. Rev. E 92, 053109.

Canaud, B., Fortin, X., Dague, N. \& Bocher, J. L. 2002 Laser MegaJoule irradiation uniformity for direct drive. Phys. Plasmas 9, 4252.

Canaud, B., Fortin, X., Garaude, F., Meyer, C. \& Philippe, F. 2004 Progress in direct-drive fusion studies for the Laser Mégajoule. Laser Part. Beams 22, 109.

CAnAud, B. \& GARAude, F. 2005 Optimization of laser-target coupling efficiency for direct drive laser fusion. Nucl. Fusion 45, L43.

Canaud, B., Garaude, F., Clique, C., Lecler, N., Masson, A., Quach, R. \& Van-der-Vliet, J. 2007 High-gain direct-drive laser fusion with indirect drive beam layout of Laser Mégajoule. Nucl. Fusion 47, 1652. 
CAnaud, B. \& Temporal, M. 2010 High-gain shock ignition of direct-drive ICF targets for the Laser Mégajoule. New J. Phys. 12, 043037.

Cavailler, C., Fleurot, N., Lonjaret, T. \& Di Nicola, J. M. 2004 Prospects and progress at LiL and MegaJoule. Plasma Phys. Control. Fusion B 46, 135.

CEA/DAM LMJ-PETAL 2020 User guide version 2.0. Available at: www-lmj.cea.fr/LMJ-PETAL-UserGroup.html.

Craxton, R. S., Anderson, K. S., Boehly, T. R., Goncharov, V. N., Harding, D. R., Knauer, J. P., Mccrory, R. L., Mckenty, P. W., Meyerhofer, D. D., Myatt, J. F., et al. 2015 Direct-drive inertial confinement fusion: a review. Phys. Plasmas 22, 110501.

Ebrardt, J. \& Chaput, M. J. 2010 LMJ on its way to fusion. J. Phys.: Conf. Ser. 244, 032017.

Frenje, J. A., Florido, R., Mancini, R., NAgayama, T., Grabowski, P. E., Rinderknecht, H., Sio, H., Zylstra, A., GAtu Johnson, M., Li, C. K., et al. 2019 Experimental validation of low-Z Ion-stopping formalisms around the Bragg peak in high-energy-density plasmas. Phys. Rev. Lett. 122, 015002.

Frenje, J. A., Grabowski, P. E., Li, C. K., Séguin, F. H., Zylstra, A. B., Gatu Johnson, M., Petrasso, R. D., Glebov, V. Y. \& SAngster, T. C. 2015 Measurements of ion stopping around the Bragg peak in high-energy-density plasmas. Phys. Rev. Lett. 11, 205001.

Gatu Johnson, M., Appelbe, B. D., Chittenden, J. P., Crilly, A., Delettrez, J., Forrast, C., Frenje, J. A., Glebov, V. Y., Grimble, W., Haines, B. M., et al. 2019 Impact of imposed mode 2 laser drive asymmetry on inertial confinement fusion implosions. Phys. Plasmas 26, 012706.

Goncharov, V. N., Betti, R., Mccrory, R. L., Sorotokin, P. \& Verdon, C. P. 1996 Self-consistent stability analysis of ablation fronts with large Froude numbers. Phys. Plasmas 3, 1402.

Gotchev, O. V., Chang, P. Y., Knauer, J. P., Meyerhofer, D. D., Polomarov, O., Frenje, J., Li, C. K., Manuel, M. J. E., Petrasso, R. D., RYGG, J. R., et al. 2009 Laser-driven magnetic-flux compression in high-energy-density plasmas. Phys. Rev. Lett. 103, 215004.

Gus'kov, S. Y., Il'in, D. V., Perlado, J. M., Rozanov, V. B., Sherman, V. E. \& Zmitrenko, N. V. 2018 Spectral composition of thermonuclear particle and recoil nuclear emissions from laser fusion targets intended for modern ignition experiments. Plasma Phys. Control. Fusion 60, 085004.

Hicks, D. G., Li, C. K., Séguin, F. H., Ram, A. K., Fremje, J. A., Petrasso, R. D., Soures, J. M., Glebov, V. Y., Meyerhofer, D. D., Roberts, S., et al. 2000 Charged-particle acceleration and energy loss in laser-produced plasmas. Phys. Plasmas 7, 5106.

Hicks, D. G., Meezan, N. B., Dewald, E. L., Mackinnon, A. J., Olson, R. E., Callahan, D. A., Doppner, T., Benedetti, L. R., Bradley, D. K., Celliers, P. M., et al. 2012 Implosion dynamics measurements at the national ignition facility. Phys. Plasmas 19, 122702.

Kugland, N. L., Ryutov, D. D., Plechaty, C., Ross, J. S. \& Park, H. S. 2012 Relation between electric and magnetic field structures and their proton-beam images. Rev. Sci. Instrum. 83, 101301.

Kull, H. J. 1991 Theory of the Rayleigh-Taylor instability. Phys. Rep. 206, 197.

Kurebayashi, S., Frenje, J. A., Séguin, F. H., Rygg, J. R., Li, C. K., Petrasso, R. D., Glebov, V. Y., Deletrez, J. A., SAngster, T. C., Meyerhofer, D. D., et al. 2005 Using nuclear data and Monte Carlo techniques to study areal density and mix in $\mathrm{D}_{2}$ implosions. Phys. Plasmas $\mathbf{1 2}$, 032703.

LI, C. K. \& Petrasso, R. D. 1993 Charged-particle stopping powers in inertial confinement fusion plasmas. Phys. Rev. Lett. 70, 3059.

Li, C. K. \& Petrasso, R. D. 2015 Erratum: charged-particle stopping powers in inertial confinement fusion plasmas. Phys. Rev. Lett. 114, 199901.

Li, C. K., Séguin, F. H., Frenje, J. A., Petrasso, R. D., Amendt, P. A., Town, R. P. J., Landen, O. L., RYGG, J. R., Betti, R., KNAUer, J. P., et al. 2009 Observations of electromagnetic fields and plasma flow in Hohlraums with proton radiography. Phys. Rev. Lett. 102, 205001.

Li, C. K., Séguin, F. H., Frenje, J. A., Rosenberg, M., Petrasso, R. D., Amendt, P. A., Koch, J. A., Landen, O. L., Park, H. S., Robey, H. F., et al. 2010 Charged-particle probing of X-ray-driven inertial-fusion implosions. Science 327, 1231. 
Li, C. K., Séguin, F. H., Frenje, J. A., Rosenberg, M. J., Rinderknecht, H. G., Zylstra, A. B., Petrasso, R. D., Amendt, P. A., Landen, O. L., Mackinnon, A., et al. 2012 Impeding Hohlraum plasma stagnation in inertial-confinement fusion. Phys. Rev. Lett. 108, 025001.

Li, C. K., Séguin, F. H., Frenje, J. A., Rygg, J. R., Petrasso, R. D., Town, R. P. J., Amendt, P. A., Hatchett, S. P., Landen, O. L., Mackinnon, A. J., et al. 2006 Monoenergetic proton backlighter for measuring $\mathrm{E}$ and $\mathrm{B}$ fields and for radiographing implosions and high-energy density plasmas. Rev. Sci. Instrum. 77 (10E), 725.

Li, C. K., Séguin, F. H., Frenje, J. A., RygG, J. R., Petrasso, R. D., Town, R. P. J., Landen, O. L., KNAUER, J. P. \& SMAlyUK, V. A. 2007 Observation of Megagauss-field topology changes due to magnetic reconnection in laser-produced plasmas. Phys. Rev. Lett. 99, 055001.

Li, C. K., Séguin, F. H., Rygg, J. R., Frenje, J. A., Manuel, M., Petrasso, R. D., Betti, R., Deletrez, J., Knauer, J. P., Marshall, F. J., et al. 2008 Monoenergetic-proton-radiography measurements of implosion dynamics in direct-drive inertial-confinement fusion. Phys. Rev. Lett. 100, 225001.

LindL, J. D. 1998 Inertial Confinement Fusion: The Quest for Ignition and high Gain using Indirect Drive. Springer.

LiOn, C. 2010 The LMJ program: an overview. J. Phys.: Conf. Ser. 244, 012003.

Manuel, M. J. E., Li, C. K., Séguin, F. H., Frenje, J., Casey, D. T., Petrasso, R. D., Hu, S. X., Betti, R., Hager, J. D., Meyerhofer, D. D., et al. 2012 First measurements of Rayleigh-Taylor-induced magnetic fields in laser-produced plasmas. Phys. Rev. Lett. 108, 255006.

Ramis, R., Canaud, B., Temporal, M., Garbett, W. J. \& Philippe, F. 2019 Analysis of three-dimensional effects in laser driven thin-shell capsule. Matter Radiat. Extremes 4, 055402.

RAMis, R. \& MEYER-TER-VEHN, J. 2016 MULTI-IFE - a one-dimensional computer code for inertial fusion energy (IFE) target simulations. Comput. Phys. Commun. 203, 226.

Ramis, R., Schmalz, R. J. \& Meyer-Ter-Vehn, J. 1988 MUlti - a computer code for one-dimensional multigroup radiation hydrodynamics. Comput. Phys. Commun. 49, 475.

Ramis, R., TEMPoral, M., CANAUd, B. \& BRANDON, V. 2014 Three-dimensional symmetry analysis of a direct-drive irradiation scheme for the Laser MegaJoule facility. Phys. Plasmas 21, 082710.

Rinderknecht, H. G., Rosenberg, M. J., Zylstra, A. B., Lahmann, B., Séguin, F. H., Frenje, J. A., Li, C. K., Gatu Johnson, M., Petrasso, R. D., Brezak Hopkins, L. F., et al. 2015 Using multiple secondary fusion products to evaluate fuel $\rho \mathrm{R}$, electron temperature, and mix in deuterium-filled implosions at the NIF. Phys. Plasmas 22, 082709.

Rosen, M. D. \& Nuckolls, J. H. 1979 Exploding pusher performance - a theoretical model. Phys. Fluids 22, 1393.

Rosenberg, M. J., Li, C. K., Fox, W., Igumenshchev, I., Séguin, F. H., Town, R. P. J., Frenje, J. A., Stoeckl, C., Glebov, V. \& Petrasso, R. D. 2015 A laboratory study of asymmetric magnetic reconnection in strongly driven plasmas. Nat. Commun. 6, 6190.

Rosenberg, M. J., Zylstra, A. B., Séguin, F. H., Rinderknecht, H. G., Frenje, J. A., Gatu Johnson, M., Sio, H., Waugh, C. J., Sinenian, N., Li, C. K., et al. 2016 A direct-drive exploding-pusher implosion as the first step in development of a monoenergetic charged-particle backlighting platform at the national ignition facility. High Energ. Dens. Phys. 18, 38.

RygG, J. R., Séguin, F. H., Li, C. K., Frenje, J. A., Manuel, M. J. E., Petrasso, R. D., Betti, R., Delettrez, J. A., GotcheV, O. V., Knauer, J. P., et al. 2008 Proton radiography of inertial fusion implosions. Science 319, 1223.

RygG, J. R., Zylstra, A. B., Séguin, F. H., Lepape, S., Bachmann, B., Craxton, R. S., Garcia, E. M., Kong, Y. Z., Gatu-Johnson, M., Khan, S. F., et al. 2015 Note: a monoenergetic proton backlighter for the national ignition facility. Rev. Sci. Instrum. 86, 116104.

Séguin, F. H., Li, C. K., Manuel, M. J. E., Rinder knecht, H. G., Sinenian, N., Frenje, J. A., RygG, J. R., Hicks, D. G., Petrasso, R. D., Delettrez, J., et al. 2012 Time evolution of filamentation and self-generated fields in the coronae of directly driven inertial-confinement fusion capsules. Phys. Plasmas 19, 012701.

Sio, H., Frenje, J. A., Le, A., Atzeni, S., Kwan, T. J. T., Gatu Johnson, M., Kagan, G., Stoeckl, C., Li, C. K., PARKer, C. E., et al. 2019 Observations of multiple nuclear reaction 
histories and fuel-ion species dynamics in shock-driven inertial confinement fusion implosions. Phys. Rev. Lett. 122, 035001.

SPITZER, L. J. JR. 1962 Physics of Fully Ionized Plasmas. Wiley Interscience.

Takabe, H., Mima, K., Montierth, L. \& Morse, R. L. 1985 Self-consistent growth rate of the Rayleigh-Taylor instability in an ablatively accelerating plasma. Phys. Fluids 28, 3676.

TEMPORAL, M. \& CANAUd, B. 2009 Numerical analysis of the irradiation uniformity of a directly driven inertial confinement fusion capsule. Eur. Phys. J. D 55, 139.

Temporal, M. \& CAnaud, B. 2011 Stochastic homogenization of the laser intensity to improve the irradiation uniformity of capsules directly driven by thousands laser beams. Eur. Phys. J. D 65, 447.

Temporal, M., Canaud, B., Garbett, W. J., Philippe, F. \& Ramis, R. 2015a Overlapping laser profiles used to mitigate the negative effects of beam uncertainties in direct-drive LMJ configurations. Eur. Phys. J. D 69, 12.

Temporal, M., Canaud, B., Garbett, W. J. \& Ramis, R. 2014a Numerical analysis of the direct drive illumination uniformity for the Laser MegaJoule facility. Phys. Plasmas 21, 012710.

Temporal, M., Canaud, B., Garbett, W. J. \& Ramis, R. 2014c Optimal laser intensity profiles for a uniform target illumination in direct drive inertial confinement fusion. High Power Laser Sci. Engng 2, e37.

Temporal, M., Canaud, B., Garbett, W. J. \& Ramis, R. 2014d Comparison between illumination model and hydrodynamic simulation for a direct drive laser irradiated target. Laser Part. Beams 32 , 549.

Temporal, M., Canaud, B., Garbett, W. J. \& Ramis, R. $2015 b$ Effect of the laser intensity profile on the shock non-uniformity in a directly driven spherical target. J. Plasma Phys. 81, 905810514.

Temporal, M., Canaud, B., Garbett, W. J. \& Ramis, R. 2015c Uniformity of spherical shock wave dynamically stabilized by two successive laser profiles in direct-drive inertial confinement fusion implosions. Phys. Plasmas 22, 102709.

Temporal, M., Canaud, B., Garbett, W. J., Ramis, R. \& Weber, S. $2014 b$ Numerical analysis of the direct Irradiation uniformity at the Laser MegaJoule facility in the context of the shock ignition scheme. High Power Laser Sci. Engng 2, e8.

Temporal, M., Ramis, R., Canaud, B., Brandon, V., Laffite, S. \& Le Garrec, B. J. 2011 Irradiation uniformity of directly driven inertial confinement fusion targets in the context of the shock-ignition scheme. Plasma Phys. Control. Fusion 53, 124008.

Zylstra, A. B., Frenje, J. A., Grabowski, P. E., Li, C. K., Collins, G. W., Fitzsimmons, P., Glenzer, S., Graziani, F., Hansen, S. B., Hu, S. X., et al. 2015 Measurement of charged-particle stopping in warm dense plasma. Phys. Rev. Lett. 114, 215002. 